Pacific Journal of Mathematics

PSEUDOCONVEX CLASSES OF FUNCTIONS. I.
PSEUDOCONCAVE AND PSEUDOCONVEX SETS 


\title{
PSEUDOCONVEX CLASSES OF FUNCTIONS I. PSEUDOCONCAVE AND PSEUDOCONVEX SETS
}

\section{ZBIGNIEW SLODKOWSKI}

\begin{abstract}
An axiomatic definition of a pseudoconvex class of functions is developed. The models include classes of subharmonic, plurisubharmonic, $q$-plurisubharmonic, convex and $q$-convex functions, and many others. Notions of dual class of functions and of pseudoconcave and pseudoconvex sets are introduced and studied. The results have applications to complex interpolation of normed spaces, given elsewhere.
\end{abstract}

Introduction. It is well known that classes of subharmonic, plurisubharmonic, and convex functions share many properties including, in particular, existence and uniqueness of the solution to the generalized Dirichlet problem. Classes of $q$-plurisubharmonic functions, studied by Hunt and Murray [4] and the author [8], follow the same pattern. During his work on [8] the author realized that most of these similarities are consequences of few simple properties.

Namely, each of these classes is a sheaf on $R^{N}$, consisting of upper semicontinuous functions with local maximum property, preserved by multiplication by positive constants. The limit of a decreasing sequence of functions of a given class belongs to it, as does supremum of several functions of this class. While only some of these classes are closed with respect to addition, all of them satisfy the following, weaker, property.

(0.1) Whenever $u$ is a function of a given class, and $v$ is a convex function, then $u+v$ is a function of the same class.

All of these classes are also translation invariant, that is $(0.2)$ whenever $u: U \rightarrow[-\infty,+\infty)$ is of given class, then $u_{y}$ is of the same class, for every $y \in R^{N}$, where $u_{y}(x)=u(x-y), x \in U+y$.

We will tentatively call any class of functions satisfying the above conditions a translation invariant pseudoconvex class on $R^{N}$. (See Examples 2.1-2.3 below.)

It turns out that considerable parts of the theory of plurisubharmonic functions, polynomially convex sets, various types of pseudoconvex and pseudoconcave sets, and some parts of potential theory 
can be generalized to the setting introduced above. In such a generalization, an arbitrary pseudoconvex class of functions is a basic object, in terms of which all the other notions (which are analogs of the classical ones) are defined. This seems to be of interest in itself, but there are still other reasons to undertake a systematic study of pseudoconvex classes of functions.

For one thing, the complete description of an arbitrary translation invariant pseudoconvex class on $R^{N}$ (which will be given in [11], a sequel to this paper) allows to easily obtain various approximation theorems for some already known pseudoconvex classes. No other proofs of these results are currently known.

Another reason, and the principal one for the author, comes from interpolation theory of normed spaces [13]. During his work [10] on generalizing the complex interpolation method of Coifman et al. [1, 2], the author realized that pseudoconvex classes of functions can serve as a natural unifying framework to study various interpolation methods. See [13] for an implementation of this program.

In this paper and its two sequels [11, 12], we study in detail pseudoconvex classes of functions. Sections 1-3 contain necessary preliminary material. In $\S 1$ we give a general definition of pseudoconvex classes which goes beyond the translation invariant case and enables one to introduce pseudoconvex classes of functions on manifolds. (In fact, we study in [12] invariant pseudoconvex classes on complex homogeneous spaces and apply the results in [13].) The crucial notion of duality between pseudoconvex classes of functions is discussed in $\S 2$. The results, that will be used in [13], are grouped in $\S \S 4$ and 5, where local maximum sets and pseudoconvex sets (with respect to a given class of functions) are studied.

\section{Discussion of axioms.}

1.1. Notation. By a class of functions on a topological space we understand a collection of functions which may be defined on different subsets of $M$. If such a class is denoted by $F$, then $F(Y)$, where $Y \subset M$, denotes the set of functions of class $F$ whose domain of definition is equal to $Y$. Typically, $F(U)$ is originally defined for $U$ open, $U \subset M$. If $Y$ is an arbitrary subset of $M$, we define $F($ nbhd $Y)=$ $\bigcup_{U \supset Y} F(U)$, where $U$ is an arbitrary open neighborhood of $Y$.

If $K$ is a compact set, $K \subset M$, we let $u \in F(K)$, if there exists a sequence $\left(u_{n}\right)_{n=1}^{\infty} \subset F($ nbhd $K)$, such that $u_{n}(x) \searrow u(x), x \in K$. If $Y$ is locally compact in $M$, we let $u \in F(Y)$, if for every compact set $K \subset Y, u \mid K \in F(K)$. 
The classes of all upper semicontinuous and continuous functions will be denoted by usc and $C$ respectively. If $P$ is any class on $M$, $C P$ denotes its intersection with $C$, i.e. $C P(U)=C(U) \cap P(U)$, if $U$ is open in $M . C P($ nbhd $Y)$ is defined in the same way as $F($ nbhd $Y$ ) above.

Definition 1.2. Let $F$ be a class of functions on a topological space $M$. We say that $F$ is a generalized pseudoconvex class of functions on $M$, if $M$ is locally compact, and conditions (1.1) through (1.6) hold. Below $U$ and $V$ denote subsets of $M$.

(1.1) $F(U) \subset \operatorname{usc}(U)$.

(1.2) Whenever $V \subset U$ and $u \in F(U)$, then $u \mid V \in F(V)$.

(1.3) Whenever $u_{n} \in F(U), u_{n}(x) \geq u_{n+1}(x), n=1,2, \ldots$, and $u(x)=\lim _{n \rightarrow \infty} u_{n}(x)$, then $u \in F(U)$.

(1.4) If $\left(u_{t}\right)_{t \in T}$ is a locally uniformly upper-bounded subfamily of $F(U)$, and $u(x)=\sup _{t \in T} u_{t}(x), x \in U$, then $u^{*} \in F(U)$, where $u^{*}$ denotes the usc regularization of $u$, i.e. $u^{*}(x)=\limsup _{y \rightarrow x} u(y)$.

(1.5) If $u \in F(U)$ and $C$ is a real number, then $(u+C) \in F(U)$.

(1.6) For every relatively compact subset $U$ of $M, F(U)$ contains a bounded function.

DEFINITION 1.3. If $F$ is a class of functions on $M$, the additive center of $F$, denoted by $A F$, is the class of all functions $v \in \operatorname{usc}(V), V$ an open subset of $M$, such that for every $u \in F(U), U$ an open subset of $M,(u+v) \in F(U \cap V)$.

Definition 1.4. A class $P$ of functions on $M$ is called pseudoconvex, if it is a generalized pseudoconvex class (in the sense of Definition 1.2) and, in addition, satisfies conditions (1.7) through (1.9).

(1.7) (Sheaf axiom). If $u \in \operatorname{usc}(U)$, where $U=\bigcup_{t \in T} U_{t}, U_{t}$ are open in $M$, and $u_{t} \mid U_{t} \in P\left(U_{t}\right)$, for $t \in T$, then $u \in P(U)$.

(1.8) (Localization axiom). Whenever $K \subset M$ is a compact set, $u \in \operatorname{usc}(K)$ and $\varepsilon>0$, then there exist $x_{0} \in K$ and $\rho \in \mathrm{AP}(\operatorname{nbhd} K)$ such that

$$
\sup _{x \in K}|\rho(x)| \leq \varepsilon, \quad(u+\rho)\left(x_{0}\right)>(u+\rho)(x), \quad x \in K \backslash\left\{x_{0}\right\} .
$$

(1.9) (Continuity axiom). Whenever $x^{*} \in M, K, L \subset M, \varphi \in C(K)$, and $\varepsilon>0$ are given, where $x^{*} \in L, L \subset \operatorname{Int}(K), K$ and $L$ are compact, then there exists a neighborhood $V$ of $x^{*}$, with $V \subset K$, such that for every $u \in P($ nbhd $K)$, satisfying inequality $u(y)<\varphi(y), y \in K$, and 
for every $x \in V$

(i) there exists a function $u_{x}^{\prime} \in P($ nbhd $L \cup V)$, such that

$$
\begin{aligned}
& u_{x}^{\prime}(y)<\varphi(y), \quad y \in L \cup V, \\
& u_{x}^{\prime}(x)>u\left(x^{*}\right)-\varepsilon
\end{aligned}
$$

(ii) there exists a function $u_{x}^{\prime \prime} \in P($ nbhd $L \cup V)$, such that

$$
\begin{aligned}
& u_{x}^{\prime \prime}(y)<\varphi(y), \quad y \in L \cup V, \\
& u_{x}^{\prime \prime}\left(x^{*}\right)>u(x)-\varepsilon .
\end{aligned}
$$

The axioms (1.8) and (1.9) may seem unclear, so we comment on them first, before discussing more systematically the definitions introduced so far.

The role of axiom (1.8) is two-fold. On one hand, in many arguments, a maximum property for some functions has to be proven. This is done by the reduction to a contradiction: with the help of (1.8), a function with a strict maximum is constructed, after which the contradiction is usually easily obtained.

On the other hand, the axiom (1.8) means also that the additive center $A P$ contains "enough" functions. For example, a sufficient condition for (1.8) to hold is that the set $A F(M)$ contains a linear subspace of functions separating points of $M$. (See the appendix.) In classical examples such a subspace is formed by linear or pluriharmonic functions.

Axiom (1.9) is an abstract analog of the property of the invariance of a class of functions $P$ with respect to the group of translations. (See Proposition 1.7 below.) It is used in those arguments in which the discontinuity of some functions of class $P$ is an obstacle. In fact, under additional assumptions, axiom (1.9) allows for local approximation of functions of class $P$ by continuous functions of the class, cf. [11, $\S 1]$.

EXAMPLE 1.5. The notions of generalized pseudoconvex and pseudoconvex classes are actually different. Define class $F$ on $\mathbf{C}$ by letting $u \in F(U), U \subset C$, if there is a locally uniformly upper bounded family $\left\{u_{t}\right\}_{t \in T}$ of harmonic functions on $U$, such that $u(x)=\sup _{t} u_{t}(x)$. One can easily see that Definition 1.2 is satisfied and (less easily) that the sheaf axiom (1.7) fails.

Definition 1.4 and the informal definition of translation invariant pseudoconvex class on $R^{N}$, given in the Introduction, differ in several respects.

On one hand, conditions (0.1) and (0.2) are changed, as they had to, since the base-space $M$ is no longer Euclidean. If $M$ has some 
geometric structure, closer analogs of conditions (0.1) and (0.2) can be given (see [12] for the case of complex homogeneous spaces), but in the abstract situation (1.8) and (1.9) seem to be their best substitutes.

On the other hand, we have also omitted from Definition 1.4 conditions (1.10) through (1.12), listed next (which are satisfied by the standard pseudoconvex classes, cf. Examples 2.1-3), although they make sense for an arbitrary base-space $M$.

(1.10) Constant functions belong to $F(U), U \subset M$.

(1.11) (Local maximum property.) For every compact set $K \subset M$, and for every $u \in F(\operatorname{nbhd} K), \max u|K \leq \max u| \partial K$.

(1.12) (Cone condition.) Whenever $u \in F(U)$ and $r$ is a positive constant, then $r u \in F(U)$.

We will comment now on conditions (1.8) through (1.12).

Note first, that condition $(0.1)$ means that in case $P$ is a translation invariant pseudoconvex class on $R^{N}$, then $A P$ contains all convex functions. Then Corollary A.2 (see Appendix) implies that axiom (1.8) holds. For another specific example of the additive center $A P$, see $[12, \S 3]$.

The practical role of condition (1.8) is that it allows for the following localization of maximum property.

Proposition 1.6. Assume that a class $F$ of functions on $M$ satisfies conditions (1.5) and (1.8). Let $K, L$ be compact sets, and $u \in \operatorname{usc}(K)$, where $L \subset K \subset M$ and $\max u|K>\max u| L$. Then there exist $x_{0} \in K \backslash L$ and $\rho \in A F($ nbhd $K)$, such that

$$
(u+\rho)\left(x_{0}\right)=0>(u+\rho)(x), \quad x \in K \backslash\left\{x_{0}\right\} .
$$

Proof. Choose $\varepsilon>0$, such that $\max u|K>2 \varepsilon+\max u| L$. Choose, by (1.8), $x_{0} \in K$, and $\rho_{1} \in A F\left(\right.$ nbhd $K$ ), such that $\sup _{x \in K}\left|\rho_{1}(x)\right|<\varepsilon$, and $\left(u+\rho_{1}\right)\left(x_{0}\right)>\left(u+\rho_{1}\right)(x), x \in K \backslash\left\{x_{0}\right\}$. Since

$$
\begin{aligned}
\max _{L}\left(u+\rho_{1}\right) & <\left(\max _{L} u\right)+\varepsilon<\left(\max _{K} u\right)-\varepsilon \\
& <\max _{K}\left(u+\rho_{1}\right)=\left(u+\rho_{1}\right)\left(x_{0}\right),
\end{aligned}
$$

we conclude that $x_{0} \notin L$. Let now $\rho(x)=\rho_{1}(x)-\left(u+\rho_{1}\right)\left(x_{0}\right)$. By (1.5), $\rho \in A F(\operatorname{nbhd} K)$, and clearly $(u+\rho)\left(x_{0}\right)=0>(u+\rho)(x)$, $x \in K \backslash\left\{x_{0}\right\}$. 
Contrary to its name, the additive center $A P$ does not have to be contained in $P$. This holds, if and only if constant functions belong to $A P$, i.e. condition (1.10) holds. See Example 1.10 below.

We will show now that translation invariant classes, $\mathrm{cf} .(0.2)$, satisfy continuity axiom (1.9).

Proposition 1.7. Let $F$ be a generalized pseudoconvex class on $M$. Let $W$ be a locally compact topological group with unit e, acting on $M$ via maps $t_{w}: M \rightarrow M, w \in W$, so that $t_{e}=\mathrm{Id}, t_{w w^{\prime}}=t_{w} \circ t_{w^{\prime}}$, and the map $(w, x) \rightarrow t_{w} x: W \times M \rightarrow M$, is continuous. Assume that the action is locally transitive in the following sense. For every $x_{0} \in M$ and for every neighborhood $W_{0}$ of $e$ in $W$, the set $\left\{t_{w} x_{0}: w \in W_{0}\right\}$ is a neighborhood of $x_{0}$ in $M$. Assume further that $F$ is invariant, i.e.

(1.13) whenever $u \in F(U)$, and $w \in W$, then $u \circ t_{w} \in F\left(t_{w}^{-1}(U)\right)$.

Then $F$ satisfies axiom (1.9).

Proof. Let $x^{*}, K, L, \varphi, \varepsilon$ be given as in (1.9). Maps $\varphi \in \mathrm{C}(K)$ and $(w, x) \rightarrow t_{w}: W \times K \rightarrow M$, are uniformly continuous, and so there is relatively compact neighborhood $W_{0}$ of $e$ such that $W_{0}^{-1}=W_{0}$, and for every $y \in K$

$$
\left\{t_{w} y: y \in L, w \in W_{0}\right\} \subset K,
$$

and the variation of $\varphi$ on $\left\{t_{w} y: w \in W_{0}\right\}$ is smaller than $\frac{1}{2} \varepsilon$. Thus

$$
u\left(t_{w} y\right)<\varphi(y)+\frac{1}{2} \varepsilon, \quad y \in K .
$$

By assumptions, the set $\left\{t_{w} x^{*}: w \in W_{0}\right\}$ contains a neighborhood of $x^{*}$; choose such a neighborhood $V$. Whenever $x \in V$, choose $w=w(x) \in W_{0}$, such that $t_{w} x^{*}=x$, and let $u_{x}^{\prime}(y)=\left(u \circ t_{w}^{-1}\right)(y)-\frac{1}{2} \varepsilon$ and $u_{x}^{\prime \prime}(y)=\left(u \circ t_{w}\right)(y)-\frac{1}{2} \varepsilon$. By (1.14), (1.13) and (1.5), functions $u_{x}^{\prime}$, $u_{x}^{\prime \prime}$ are of class $F$ on a neighborhood of $L$. The inequalities required in (1.9) (i), (ii) hold by (1.15).

The last proposition goes beyond the Euclidean case and is sufficient for most purposes, cf. [12]. However, if $M$ is, e.g. a closed unit ball in $\mathbf{C}^{n}$, then the Möbius group acts continuously on $M$, but its action is transitive on the open ball only. Lemma 1.9 helps to handle such situations.

Definition 1.8. Let $F$ be a class of functions on $M$ and $x \in M$. We say that $x$ is a peak for $F$ if, given a compact set $K$ with $x \in K$, 
a neighborhood $U$ of $x$ and $E<0$, there is $u \in F(\operatorname{nbhd} K)$, such that

$$
\begin{aligned}
& u|K \leq 0, \quad u| K \backslash U \leq E \\
& \lim _{y \rightarrow x} u(y)=u(x)=0 .
\end{aligned}
$$

LEMMA 1.9. Let $P$ be a pseudoconvex class on $M$. Let $\Gamma$ be a closed subset of $M$ consisting of peak points for $P$. Assume that class $P$ satisfies continuity axiom (1.9) for subsets $K \subset M \backslash \Gamma$. Then $P$ satisfies (1.9) on $M$.

Proof. Let $x^{*}, L, K, \varphi, \varepsilon$ be as in (1.9).

Case $x^{*} \notin \Gamma$. Using (1.5) and Definition 1.8, we find for each $z \in \Gamma$ a function $h_{z} \in F($ nbhd $K)$ and a neighborhood $M_{z}$ of $z$, such that

$$
\begin{aligned}
& h_{z}(y)<\varphi, \quad y \in K, \\
& h_{z}(y)>\varphi(y)-\frac{1}{2} \varepsilon, \quad y \in M_{z} .
\end{aligned}
$$

Choose from $\left\{M_{z}\right\}$ a finite covering $M_{z(1)}, \ldots, M_{z(n)}$, of $K \cap \Gamma$, which we denote further by $M_{1}, \ldots, M_{n}$, and let $h_{1}, \ldots, h_{n}$ denote the corresponding functions $h_{z(1)}, \ldots, h_{z(n)}$. Let $M=M_{1} \cup \cdots \cup M_{n}$ and $h(x)=\max \left(h_{1}(x), \ldots, h_{n}(x)\right)$. Choose compact subsets $L_{0}, K_{0}$, of $L$ and $K$ respectively, so that $L_{0} \subset \operatorname{Int}\left(K_{0}\right), K_{0} \cap \Gamma=\varnothing, L \subset L_{0} \cup M$ and $K \subset K_{0} \cup M$.

Since axiom (1.9) holds on $M \backslash \Gamma$, there is a neighborhood $V$ of $x^{*}$, such that $V \subset M \backslash \Gamma, \bar{V} \subset$ Domain of $f$, and for every $x \in V$ there are functions $u_{x}^{1}, u_{x}^{2} \in P\left(\mathrm{nbhd} K_{0}\right)$, such that

$$
\begin{aligned}
& u_{x}^{1}<\varphi-\frac{1}{2} \varepsilon, \quad u_{x}^{2}<\varphi-\frac{1}{2} \varepsilon, \quad \text { on } L_{0} \cup \bar{V}, \\
& u_{x}^{1}(x)>u\left(x^{*}\right)-\varepsilon, \quad u_{x}^{2}\left(x^{*}\right)>u(x)-\varepsilon .
\end{aligned}
$$

Define now

$$
\begin{aligned}
& u_{x}^{\prime}(y)= \begin{cases}\max \left(u_{x}^{1}(y), h(y)\right), & y \in \operatorname{nbhd}\left(L_{0} \cup \bar{V}\right), \\
h(y), & y \in M \backslash\left(L_{0} \cup \bar{V}\right) ;\end{cases} \\
& u_{x}^{\prime \prime}(y)= \begin{cases}\max \left(u_{x}^{2}(y), h(y)\right), & y \in \operatorname{nbhd}\left(L_{0} \cup \bar{V}\right), \\
h(y), & y \in M \backslash\left(L_{0} \cup \bar{V}\right) .\end{cases}
\end{aligned}
$$

By (1.4) $h \in P(M)$ and $u_{x}^{\prime}, u_{x}^{\prime \prime} \in P\left(\operatorname{nbhd}\left(L_{0} \cup \bar{M}\right)\right)$. By $(1.19), h(y)>$ $\varphi(y)-\frac{1}{2} \varepsilon$, and so by (1.20), the definitions of $u_{x}^{\prime}, u_{x}^{\prime \prime}$ are consistent, provided $\operatorname{nbhd}\left(L_{0} \cup \bar{V}\right)$ is chosen small enough. By sheaf axiom (1.7) 
$u_{x}^{\prime}, u_{x}^{\prime \prime}$ are of class $P$ on $\operatorname{nbhd}\left(L_{0} \cup \bar{V}\right) \cup M$, which contains $L$. The remaining properties of $u_{x}^{\prime}, u_{x}^{\prime \prime}$ are obvious.

Case $x^{*} \in \Gamma$. Using Definition 1.8, we choose a neighborhood $V$ of $x^{*}$, and $h \in P($ nbhd $K)$, such that $\left|\varphi(y)-\varphi\left(x^{*}\right)\right|<\frac{1}{2} \varepsilon, y \in V, h<\varphi$ on $K$, and $h(y)>\varphi(y)-\frac{1}{2} \varepsilon, y \in V$. Then let $u_{x}^{\prime} \equiv h, u_{x}^{\prime \prime} \equiv h$. It is clear that $V, u_{x}^{\prime}, u_{x}^{\prime \prime}$ satisfy the requirements of (1.9).

Although we did not include (1.10)-(1.12) into the axioms of a pseudoconvex class, we will still have to assume them occasionally, while studying some topics. For example, (1.12) is assumed throughout [13, 14].

In Definition 1.4 , the role of condition (1.10) is partly played by axiom (1.6).

EXAMPLE 1.10. Let $L$ be a fixed real parameter. Define class $C_{L}^{1}$ on $R^{N}$ as in [8, §2]. Namely, $u \in C_{L}^{1}(U)$, if the function $v(x)=$ $u(x)+\frac{1}{2} L|x|^{2}$ is locally convex on $U$. By [8, Proposition 2.3], class $C_{L}^{1}$ satisfies axioms (1.1)-(1.8), and (0.2), and so is pseudoconvex by Proposition 1.7.

Clearly, $C_{L}^{1}$ contains constant functions, if and only if $L \geq 0$. (In contrast, it always contains a locally bounded function $x \rightarrow-\frac{1}{2} L|x|^{2}$. The last example shows also that local maximum property does not hold for an arbitrary pseudoconvex class. Namely, $C_{L}^{1}$ satisfies (1.11), if and only if $L \leq 0$. These two observations suggest that conditions (1.10), (1.11) might be related by some kind of duality. The next definition and Proposition 1.12 confirm this.

DEFINITION 1.11 . Let $F$ be a class of usc functions on a locally compact space $M$. The dual class to $F$, denoted $F^{\mathrm{d}}$, consists of functions $u \in \operatorname{usc}(U), U \subset M, U$ open, such that for every $f \in F(V), V \subset M$, $V$ open, the function $u+f$ has local maximum property (1.11).

Although property (1.11) may fail for a pseudoconvex class $P$, it still holds for the class $P+P^{\mathrm{d}}$. Through this duality, the local maximum property is still important in the study of general pseudoconvex classes.

Proposition 1.12. Let $F$ be a generalized pseudoconvex class on M. Then

(i) $F$ has local maximum property, if and only if $F^{\mathrm{d}}$ contains constant functions;

(ii) if $F^{\mathrm{d}}$ contains constant functions, then $F$ has local maximum property. 
This proposition follows immediately from (1.5) and definitions.

The study of duality is continued in $\S 2$.

REMARK 1.13. Property (1.12) is very natural and simplifies many arguments, cf. [13]. On the other hand, classes $C_{L}^{1}$, for $L<0$, do not have it, as well as the one defined next.

EXAMPLE. Let $u \in P(U), U$ open in $R^{N}$, if the distributional Laplacian of $u$ is a measure greater than or equal to the Lebesgue measure.

These examples suggest that pseudoconvex classes (without cone condition) might be an appropriate setting to study nonhomogeneous Dirichlet problems (e.g. nonhomogeneous Monge-Ampere equations).

The next proposition shows that, under marginal exceptions, cone condition (1.12) implies both (1.10) and (1.11).

Proposition 1.14. Let $F$ be a generalized pseudoconvex class on $M$ satisfying (1.12). Then

(i) $F^{\mathrm{d}}$ satisfies cone condition (1.12);

(ii) $F$ contains constant functions;

(iii) $F$ has local maximum property (1.11), provided $F$ satisfies axiom (1.8), and for every $x \in M$, there is $u \in F^{\mathrm{d}}(\operatorname{nbhd} x)$, such that $u(x) \neq-\infty$

(iv) if $u \in F(U), U \subset M$, and $g: R \rightarrow R$ is an increasing convex function, then $g \circ u \in F(U)$.

Proof. (i) Obvious, by Definition (1.11).

(ii) Let $U \subset M$ be open and relatively compact. By (1.6), there is a bounded function $u_{0} \in F(U)$. Let $u(x)=u_{0}(x)+\sup _{x \in U}|u(x)|$. By (1.5), $u \in F(U)$. By condition (1.12), $n^{-1} u \in F(U), n=1,2, \ldots$ Since $n^{-1} u(x) \searrow 0$ (note $u(x) \geq 0$ ), axiom (1.3) implies that the constant function 0 belongs to $F(U)$.

(iii) Suppose $F$ does not have local maximum property (1.11). Then there is a compact set $K$, and $f \in F(\operatorname{nbhd} K)$, such that $\max f \mid K>$ $\max f \mid \partial K$. By Proposition 1.6, there is a function $\rho \in A F(\operatorname{nbhd} K)$, and $x_{0} \in \operatorname{Int}(K)$, such that $(f+\rho)\left(x_{0}\right)=0>(f+\rho)(x), x \in K \backslash\left\{x_{0}\right\}$. By assumption (iii), there is a relatively compact neighborhood $V$ of $x_{0}$ and $u \in F^{\mathrm{d}}($ nbhd $\bar{V})$, such that $\bar{V} \subset K$, and $u\left(x_{0}\right)>-\infty$. Choose $\varepsilon>0$, so that, if $g=f+\rho+\varepsilon$, then

$$
g\left(x_{0}\right)>0>\max g \mid \partial V .
$$


Choose $r>0$ so that $r g\left(x_{0}\right)+u\left(x_{0}\right)>\max u \mid \bar{V}$. By this and (1.21), we get $(r g+u)\left(x_{0}\right)>\max (r g+u) \mid \partial V$. This is a contradiction, since $(r g+u) \in r(F+A F)+F^{\mathrm{d}} \subset F+F^{\mathrm{d}}$.

(iv) Since $g$ is convex and increasing, $g(x)=\sup _{t \in T} l_{t}(x)$, where $l_{t}(x)=a_{t}+b_{t} x, b_{t}>0$. Thus, $(g \circ u)(x)=\sup _{t \in T}\left(a_{t}+b_{t} u(x)\right)$. By (1.12), $\left(b_{t} u\right) \in F(U)$, and by (1.5), $\left(a_{t}+b_{t} u\right) \in F(U)$. Since $g \circ u$ is already usc, $g \circ u=\sup l_{t} \circ u$ belongs to $F(U)$ by axiom (1.4).

2. Duality. Many pseudoconvex classes can be represented as dual, in the sense of Definition 1.11, to some simpler ones. We will show in [11] that every proper translation invariant pseudoconvex class $P$ on $R^{N}$ is dual to a class $F$, where $F$ can be chosen as a class of smooth functions (in fact, as a class of quadratic polynomials). We will give now several examples of such representations.

EXAMPLE 2.1. $P=$ the class of all subharmonic functions on open subsets of $R^{N}$. Then $P=F_{1}^{\mathrm{d}}$ and $P=F_{2}^{\mathrm{d}}$, where $F_{1}=$ the class of all harmonic functions and $F_{2}=$ the class of all quadratic harmonic polynomials. In fact, $P=P^{\mathrm{d}}$, i.e. $P$ is self-dual.

EXAMPLE 2.2. $P=P_{q}$, the class of all $q$-plurisubharmonic functions on $\mathbf{C}^{n}$, where $0 \leq q \leq n-1$, cf. [4], [8]. Then $P_{q}=F^{\mathrm{d}}$, where $F=$ the class of all smooth functions whose complex Hessian has at least $(n-q)$ nonnegative eigenvalues (at every point); cf. [8, Proposition 1.1]. Also, $P_{q}=F_{1}^{\mathrm{d}}$, where $F_{1}=$ the set of all functions $f$, such that $f \mid L$ is plurisubharmonic, $f \mid \mathbf{C}^{n} \backslash L \equiv-\infty$, for some hyperplane of complex dimension $(q+1)$ in $\mathbf{C}^{n}$.

EXAMPLe 2.3. $P=$ the class of all $q$-convex functions on $R^{N}$. Here $P=F^{\mathrm{d}}$, where $F=$ the collection of all functions $f$ on $R^{N}$, such that $f \mid L$ is convex, $f \mid R^{N} \backslash L \equiv-\infty$, for some $(q+1)$-dimensional hyperplane $L$ in $R^{N}$.

Similar representations can be given for pseudoconvex classes $\psi(q)$, $q=1,2 \ldots$, introduced by $\mathrm{Wu}[16, \S 1]$. Further examples of dual pseudoconvex classes are given in $[\mathbf{1 1}, \mathbf{1 2}]$.

Proposition 2.4. A class $P$ of functions on a locally compact space $M$ is dual to some class $F$ of usc functions on $M$, if and only if it is equal to its own bidual i.e. $P=P^{\mathrm{dd}}$.

Proof. The sufficiency is obvious: $P=F^{\mathrm{d}}$, where $F=P^{\mathrm{d}}$. 
Necessity. If $P=F^{\mathrm{d}}$, then all the classes $F+F^{\mathrm{d}}, F^{\mathrm{dd}}+F^{\mathrm{d}}$ and $F^{\text {dd }}+F^{\text {ddd }}$ have the local maximum property, by Definition 1.11 . Since $F^{\text {ddd }}$ is the largest of all classes $F_{1}$, such that $F^{\text {dd }}+F_{1}$ has local maximum property, it follows that $F^{\text {ddd }}$ contains $F_{1}=F^{\text {d }}$. In the same way $F^{\text {dd }}$ contains $F$, and so $F+F^{\text {ddd }}$ has local maximum property. Similarly, $F^{\mathrm{d}}$ is the largest of all classes $F_{2}$, such that $F+F_{2}$ has local maximum property, and so $F^{\mathrm{d}}$ contains $F_{2}=F^{\mathrm{ddd}}$. Thus, $F^{\mathrm{d}}=F^{\mathrm{ddd}}$, i.e. $P=P^{\mathrm{dd}}$.

We prove now that non-triviality of $F^{\mathrm{d}}$ implies weaker forms of maximum property for the class $F$ itself.

Proposition 2.5. Let $F$ be a class of usc functions on a locally compact space $M$. Let $U \subset M$ be open. Then

(i) if $F^{\mathrm{d}}(U)$ contains a bounded function, then there exists a constant $C$, depending only on $U$, such that for every compact $K \subset U$ and for every function $u \in F($ nbhd $K$ ),

$$
\max u \mid K \leq C+\max (u \mid \partial K) .
$$

(ii) if $x \in U$, and there exists a function $g \in F^{\mathrm{d}}(U)$, such that $g(x)>-\infty$, then there exists a constant $C(x)$, depending only on $x$ and $U$, such that for every compact $K \subset U$ and for every $u \in F($ nbhd $K$ )

$$
u(x) \leq C(x)+\max (u \mid \partial K) .
$$

Proof. If $g \in F^{\mathrm{d}}(U)$, then for every $u, K$ as above $\max (u+g) \mid K \leq$ $\max (u+g) \mid \partial K$, by the definition of $F^{\mathrm{d}}$. Hence, for $x \in K$,

$$
\begin{aligned}
u(x) & \leq \max (u+g) \mid \partial K-g(x) \\
& \leq(-g(x)+\max g \mid \partial K)+\max u \mid \partial K .
\end{aligned}
$$

If $g(x)>-\infty$, as in (ii), we let $C(x)=-g(x)+\max g \mid \partial U$, and (2.2) is proved.

If $g$ is bounded, as in (i), then let $C=\max (-g)|K+\max g| \partial K$, and (2.2) holds with this value of $C$, by (2.3).

The next two remarks follow immediately from Definitions 1.11 and 1.3.

REMARK 2.6. Let $F$ be a class of usc functions on $M$ invariant with respect to homeomorphisms $t_{w}: M \rightarrow M, w \in W$, in the sense of (1.13). Then $F^{\mathrm{d}}$ is invariant with respect to $t_{w}, w \in W$. 
REMARK 2.7. If $F$ is a class of usc functions on $M$, then $A F \subset$ $A\left(F^{\mathrm{d}}\right)$.

The following lemma is a local criterion for a function to belong to the dual class.

LEMMA 2.8. Let $F$ be a class of usc functions on a locally compact space $M$, such that (1.5) and (1.8) hold. Let $u \in \operatorname{usc}(U)$, $U$ open in $M$. Then $u$ does not belong to $F^{\mathrm{d}}(U)$, if and only if there are a point $x \in U$, a neighborhood $V$ of $x, V \subset U$, and $u_{0} \in F(V)$, such that

$$
\left(u+u_{0}\right)(x)=0>\left(u+u_{0}\right)(y), \quad y \in V \backslash\{x\} .
$$

Proof. The sufficiency is obvious; as for the necessity, if $u \notin F^{\mathrm{d}}(U)$, then there is $u_{1} \in F\left(U_{1}\right)$ and a compact $K \subset U \cap U_{1}$, such that

$$
\max \left(u_{1}+u\right)\left|K>\max \left(u_{1}+u\right)\right| \partial K \text {. }
$$

By Proposition 1.6, there is $v \in A F(\operatorname{nbhd} K)$ and $x_{0} \in \operatorname{Int}(K)$, such that

$$
\left(u_{1}+u\right)(x)+v(x)=0>\max \left(\left(u_{1}+u\right)+v\right)(y), \quad y \in K \backslash\{x\} .
$$

Let $u_{0}=u_{1}+v$ and $V=\operatorname{Int}(K)$. Then $u_{0} \in F(V)$ and (2.4) holds.

It is natural to ask whether the dual and bidual of a pseudoconvex class $P$ must be pseudoconvex as well, and if so, whether $P=P^{\mathrm{dd}}$. We will prove in [11] that it is indeed so in the translation invariant case. Below, we give partial results in this direction in the general case.

LEMMA 2.9. Let $F$ be a class of usc functions on a locally compact space $M$.

(i) Assume that axiom (1.5) holds for $F$. Then, the dual class $P=F^{\mathrm{d}}$ satisfies axioms (1.1)-(1.3), (1.5), and the following weaker form of (1.4):

whenever $u \in \operatorname{usc}(U), U \subset M$ and $u(x)=\sup _{t} u_{t}(x), x \in$ $U$, where $\left\{u_{t}\right\}_{t \in T} \subset P(U)$, then $u \in P(U)$.

(ii) Assume that axiom (1.8) holds for $F$. Then, $P=F^{\mathrm{d}}$ satisfies (1.7), (1.8) and the following "minorant property":

(2.5) if $u \in \operatorname{usc}(U)$ is such that for every $x \in U$ there exists $u_{x} \in P\left(U_{x}\right), U_{x}$ open, $x \in U_{x} \subset U$, with $u_{x}(x)=$ $u(x), u_{x}(y) \leq u(y), y \in U_{x}$, then $u \in P(U)$. 
LEMMA 2.10. Let $F$ be a class of usc functions on a locally compact space $M$, satisfying axioms (1.5) and (1.9)(i). Assume that (1.6) holds for $F^{\mathrm{d}}$. Then, $P=F^{\mathrm{d}}$ is a generalized pseudoconvex class.

REMARK. The assumption on $F^{\mathrm{d}}$ is probably too strong but it cannot just be omitted: if $F=$ usc, then $F^{\text {d }}$ consists only of the constant function $\equiv-\infty$.

Corollary 2.11. Let $P$ be a pseudoconvex class of functions on $M$ invariant with respect to a continuous and locally transitive action of a locally compact group $W$ on $M$ (as in Proposition 1.7). If $P^{\mathrm{d}}(U)$ contains a bounded function whenever $U$ is relatively compact, then both $P^{\mathrm{d}}$ and $P^{\mathrm{dd}}$ are pseudoconvex classes on $M$.

Proof. Follows immediately from Proposition 1.7, Remark 2.6 and Lemmas 2.9, 2.10.

Proof of Lemma 2.9(i). It is obvious, by Definition 1.11, that conditions (1.1), (1.2), (1.5) hold for $F^{\mathrm{d}}$. To check (1.3), take $u_{n} \in F^{\mathrm{d}}(U)$, with $u_{n}(x) \geq u_{n+1}(x), n=1,2, \ldots$, and $u_{n}(x) \searrow u(x), x \in U$, and consider $f \in F(V)$. Then, the functions $w_{n}=u_{n}+f$ have local maximum property and $w_{n}(x) \searrow u(x)+f(x), x \in U$. Thus, $u+f$ has local maximum property for every $f \in F(U)$, and so $u \in F^{\mathrm{d}}(U)$.

As for the weaker form of (1.4), if $u$ and $u_{t}, t \in T$, are as in Lemma 2.9(i), take $f \in F(V)$ and let $w_{t}=u_{t}+f$. Then, $\sup _{t} w_{t}(x)=$ $u(x)+f(x), x \in V \cap U$, and so $u+f$ has local maximum property for every $f \in F$. Consequently, $u \in F^{\mathrm{d}}(U)$ ( $u$ is usc by assumption), as required.

(ii) By Remark 2.7, class $F^{\mathrm{d}}$ satisfies axiom (1.8). The sheaf condition (1.7) is a special case of property (2.5).

Suppose (2.5) fails. Then, by Lemma 2.8, there are a point $x$, a neighborhood $V$ of $x$ and $u_{0} \in F(V)$, such that inequality (2.4) holds. If $U_{x}$ and $u_{x} \in P\left(U_{x}\right)$ are as in condition (2.5), then, by (2.4),

$$
\begin{array}{r}
\left(u_{x}+u_{0}\right)(x)=u(x)+u_{0}(x)=0>\left(u+u_{0}\right)(x) \geq u_{x}(y)+u_{0}(y), \\
\text { for } y \in U_{x} \backslash\{x\} .
\end{array}
$$

Thus, $\left(u_{x}+u_{0}\right)$ has strict local maximum at $x$, which contradicts the assumption that $\left(u_{x}+u_{0}\right) \in F^{\mathrm{d}}+F$. 
Proof of Lemma 2.10. Note that, in the last proof, Condition (1.8) was used only to prove (2.5) and (1.7). So, it remains to show that axiom (1.4) holds for $F^{\mathrm{d}}$.

Let $u_{t}, t \in T, u$ and $u^{*}$ be as in (1.4). Suppose, $u^{*} \notin F^{\mathrm{d}}(U)$. By Definition 1.11, there are a compact $L \subset U, x_{0} \in \operatorname{Int}(L)$ and $f \in F($ nbhd $L)$, such that

$$
u^{*}\left(x_{0}\right)+f\left(x_{0}\right)>\max \left(u^{*}+f\right) \mid \partial L .
$$

Choose a compact $K \subset U$, such that $L \subset \operatorname{Int}(K)$ and $K \subset \operatorname{Dom}(f)$. Choose $\varepsilon>0$ and $\varphi \in C(K)$, such that $\varphi(y)>f(y), y \in K$, and $u^{*}\left(x_{0}\right)+f\left(x_{0}\right)>2 \varepsilon+\max \left(u^{*}+\varphi\right) \mid \partial L$. We now apply axiom (1.9)(i) to the data $x_{0}, K, L, \varphi, \varepsilon$ and get a neighborhood $V$ of $x_{0}$ with properties postulated therein. Without loss of generality, $V \subset L$.

By the definition of usc envelope $u^{*}$, there is $x \in V$ and $t(x) \in T$, such that $u_{t(x)}(x)>u^{*}\left(x_{0}\right)-\varepsilon$. We now apply part (i) of axiom (1.9) to the function $f<\varphi$, and obtain $f_{x}^{\prime} \in F$ (nbhd $L$ ), such that $f^{\prime}(y)<\varphi(y), y \in K$, and $f_{x}^{\prime}(x)>f\left(x_{0}\right)-\varepsilon$. By these inequalities and (2.6), we get

$$
\begin{aligned}
\left(u_{t(x)}+f_{x}^{\prime}\right)(x) & >u^{*}\left(x_{0}\right)-\varepsilon+f\left(x_{0}\right)-\varepsilon>\max \left(u^{*}+\varphi\right) \mid \partial L \\
& >\max \left(u_{t(x)}+f_{x}^{\prime}(x)\right) \mid \partial L .
\end{aligned}
$$

This contradicts the local maximum property of the function $\left(u_{t(x)}+f_{x}^{\prime}\right) \in F^{\mathrm{d}}+F$.

3. Constructing new functions of class $P$. As shown in Lemma 2.9, the dual class $P=F^{\mathrm{d}}$ has the "minorant property" $(2.5)$ which is (at least formally) stronger, than the sheaf axiom (1.7). It is open, whether the minorant property holds for an arbitrary pseudoconvex class but it will be proved in [11] that it does hold for all translation invariant pseudoconvex classes. In this section, we discuss some weaker versions of property (2.5).

Other methods of producing functions of class $P$ are related to the Dirichlet problem and will be studied in a subsequent paper.

REMARK 3.1. The minorant property (2.5) implies the following

(3.1) (Reiteration property). If $U_{1} \subset U \subset M, U_{1}, U$ open, and $u_{1} \in P\left(U_{1}\right), u \in P(U)$ are such that $\limsup _{y \rightarrow x} u_{1}(y) \leq u(x)$, for $x \in U \cap\left(\partial U_{1}\right)$, then $v \in P(U)$, where

$$
v(x)= \begin{cases}\max \left(u_{1}(x), u(x)\right), & x \in U_{1}, \\ u(x), & x \in U \backslash U_{1} .\end{cases}
$$


In particular, the dual class $P=F^{\mathrm{d}}$ in Lemma 2.9 has property (3.1).

Proof. To apply (2.5), we let $v_{x} \equiv u_{1}$ and $U_{x}=U_{1}$, in case $u_{1}(x)>$ $u(x)$, and $v_{x} \equiv u, U_{x}=U$ otherwise. The assumptions of (2.5) being satisfied, $v \in P(U)$.

Proposition 3.2. Let $F$ be a generalized pseudoconvex class on $M$ and $\left(u_{n}\right) \subset F(U), U \subset M$. If $u_{n}$ converges uniformly to $u$ on $U$, then $u \in F(U)$.

Proof. Choose a subsequence $n(k), k=1,2, \ldots$, such that $\sup \left|u_{n(k)}(x)-u(x)\right| \leq 2^{-k-2}$. Let $v_{k}(x)=2^{-k}+u_{n(k)}(x)$. Then, $v_{k}(x) \geq v_{k+1}(x), k=1,2, \ldots$, and so $v_{k}(x) \searrow v(x), x \in U$. By (1.5), $v_{k} \in F(U)$ and by (1.3), $u \in F(U)$.

Proposition 3.3. Let $P$ be a pseudoconvex class on $M$. Then, the reiteration property (3.1) holds, provided function $u$ in (3.1) is continuous at the point of $U \cap\left(\partial U_{1}\right)$.

Proof. In the notation of (3.1), let, for $n=1,2, \ldots$,

$$
v_{n}(x)= \begin{cases}\max \left(u_{1}(x), u(x)+n^{-1}\right), & x \in U_{1}, \\ u(x)+n^{-1}, & x \in U \backslash U_{1} .\end{cases}
$$

We will show first that $v_{n} \in P(U)$ by checking that the sheaf axiom (1.7) holds, i.e. every $x \in U$ has a neighborhood $U_{x}$, such that $v_{n} \mid U_{x} \in$ $P\left(U_{x}\right)$. If $x \in U_{1}$, take $U_{x}=U_{1}$ and, if $x \in U \backslash U_{1}$, then $U_{x}=U \backslash \bar{U}_{1}$ works. If $x \in U \cap\left(\partial U_{1}\right)$, then, by the continuity of $u$ at $x$, there is a neighborhood $U_{x}$ of $x$, such that $u_{1}<u+n^{-1}$ on $U_{x}$, and so $v_{n} \mid U_{x}=\left(u+n^{-1}\right) \in P\left(U_{x}\right)$.

Since $v_{n} \in P(U), n=1,2, \ldots$, and $v_{n}(x) \searrow v(x), x \in U$, function $v$ must be of class $P$.

Recall that a covering $\left\{V_{t}\right\}_{t \in T}$ is locally finite, if every point $x$ has a neighborhood $U_{x}$, which intersects only finitely many $V_{t}$ 's.

COROLlary 3.4. Let $P$ be a pseudoconvex class of functions on $M$, and $\left\{V_{t}\right\}_{t \in T}$ be a locally finite open covering of an open set $V \subset M$. Let $u \in C(V), v_{t} \in C\left(\bar{V}_{t}\right), t \in T$. Assume that $v_{t} \mid V_{t} \in P\left(V_{t}\right)$ and

$$
v_{t}\left|\partial V_{t}=u\right| \partial V_{t},
$$




$$
u \mid \bar{V}_{t} \leq v_{t}
$$

Let $v(x)=\max \left\{v_{t}(x): x \in V_{t}\right\}, x \in V$. Then, $v \in P(V) \cap \mathrm{C}(V)$.

Proof. Obviously, $v$ is continuous.

Assertion. If $v_{i} \in C\left(\bar{V}_{i}\right), v_{i} \mid V_{i} \in P\left(V_{i}\right), i=1,2$, and $v_{i} \mid\left(\partial V_{i}\right) \cap$ $V_{j} \leq v_{j} \mid\left(\partial V_{i} \cap V_{j}\right)$, where $\{i, j\}=\{1,2\}$, then $v \in C\left(\bar{V}_{1} \cup \bar{V}_{2}\right)$ and $v \mid\left(V_{1} \cup V_{2}\right) \in P$, where $v(x)=\max \left(v_{1}(x), v_{2}(x)\right), x \in \bar{V}_{1} \cap \bar{V}_{2}, v(x)$ $=v_{1}(x), x \in \bar{V}_{1} \backslash \bar{V}_{2}, v(x)=v_{2}(x), x \in \bar{V}_{2} \backslash \bar{V}_{1}$.

Indeed, if $T=\{1,2\}$, then $V=V_{1} \cup V_{2}$ and, by (1.7), it suffices to show that $v \mid V_{i} \in P\left(V_{i}\right), i=1$, 2. To show $v \mid V_{1} \in P\left(V_{1}\right)$, we apply reiteration property (3.1) (Proposition 3.3) to the data $U:=V_{1}, U_{1}:=$ $V_{1} \cap V_{2}, u=v_{1}\left|V_{1}, u_{1}=v_{2}\right| V_{1} \cap V_{2}$. Then, $v(x)=\max \left(u_{1}(x), u(x)\right)$, $x \in U_{1}$ and $v(x)=u(x), x \in U \backslash U_{1}$. The required assumptions hold by (3.2), (3.3), and so $v \mid V_{1} \in P\left(V_{1}\right)$. By similar argument for $i=2$, $v \in P(V)$.

Consider now a sequence $\left(V_{n}\right)$ of sets from the covering $\left\{V_{t}\right\}$ with corresponding functions $v_{n} \in P\left(V_{n}\right)$ and let $V^{(n)}=V_{1} \cup \cdots \cup V_{n}$ and $v^{(n)}(x)=\max \left\{v_{i}(x): x \in V_{i}\right\}$. One checks easily that the pairs $V^{(n)}$, $V_{n+1}$, and $v^{(n)}, v_{n+1}$, satisfy the assumption of the Assertion, and that $v^{(n+1)}=\max \left(v^{(n)}, v_{n+1}\right)$ in $V^{(n)} \cap V_{n+1}, v^{(n+1)}=v^{(n)}$ in $V^{(n)} \backslash V_{n+1}$ and $v^{(n+1)}=v_{n+1}$ in $V_{n+1} \backslash V^{(n)}$. Applying inductively the Assertion, we get $v^{(n)} \in C P\left(V_{n}\right), n=1,2, \ldots$.

If $U$ is relatively compact in $V$, sequence $\left(V_{n}\right)$ covers $\bar{U}$, and if $n$ is large enough, then $v\left|U=v^{(n)}\right| U$, and so, by (1.7), $v \in P(V)$.

THEOREM 3.5. Let $P$ be a pseudoconvex class on $M$ and $u \in C(U)$, where $U$ is open in $M$. Assume that for every $x \in U$, there are $a$ neighborhood $U_{x}$ of $x$ and a continuous function $u_{x} \in P\left(U_{x}\right)$, such that

$$
u_{x}(x)=u(x), \quad u_{x}(y)<u(y), \quad y \in U_{x} \backslash\{x\} .
$$

Then $u \in P(U)$.

Proof. Fix $\varepsilon>0$ and choose for every $x \in U$ a positive number $\varepsilon(x)<\varepsilon$, such that the open set $V_{x}=\left\{y \in U: v_{x}(y)>u(y)-\varepsilon(x)\right\}$ is relatively compact in $U_{x}$.

By (1.7), it suffices to show that $u \mid H \in P(H)$ whenever $H$ is open and relatively compact in $U$. Fix such $H$ and choose a finite covering of $\bar{H}$ by sets $V_{x(1)}, \ldots, V_{x(n)}$. Index these neighborhoods as $V_{1}, \ldots, V_{n}$, 
the corresponding functions $u_{x}$ as $u_{1}, \ldots, u_{n}$, and $\varepsilon(x(j))$ as $\varepsilon_{j}, j=$ $1, \ldots, n$. Let $v_{j}(y)=u_{j}(y)+\varepsilon_{j}, y \in V_{j}, j=1, \ldots, n$, and $V=$ $V_{1} \cup \cdots \cup V_{n}$. Clearly, the functions $v_{1}, v_{2}, \ldots, v_{n}, u$, and the covering $\left\{V_{1}, \ldots, V_{n}\right\}$ of $V$ satisfy the assumptions of Corollary 3.4, and so $v \in P(V)$, where $v(x)=\max \left\{v_{j}(x): x \in V_{j}\right\}$.

On the other hand, $u(x) \leq v(x) \leq u(x)+\varepsilon, x \in V$. Let $v_{\varepsilon}=v \mid H$. We have constructed for every $\varepsilon>0$ a function $v_{\varepsilon} \in P(H)$, such that $\left\|v_{\varepsilon}-(u \mid H)\right\|_{\infty}<\varepsilon$. By Proposition 3.2, $u \in P(H)$.

4. Sets with local maximum property and saturations. In this section we study systematically local maximum property for sets and the notion of saturation of a set (Definition 4.11), which is an analog of the Perron envelope in this context. The notion of saturation is crucial for our construction of interpolation spaces in [13].

DEFINITION 4.1. Let $F$ be a class of functions on a locally compact space $M$. A subset $X$ of $M$ is called an $F$-maximum set, if $X$ is locally compact (i.e. $X=\bar{X} \cap V$ for some open subset $V$ of $M$ ), and for every compact $K \subset M$, such that $K \cap X$ is compact, and for every $f \in F(\operatorname{nbhd}(K \cap X))$

$$
\max f|K \cap X \leq \max f|(\partial K) \cap X .
$$

If $M=C^{n}$ and $F=$ the class of all plurisubharmonic functions on $C^{n}(n>1)$, the most natural examples of $F$-maximum sets are complex analytic varieties without isolated points. Other examples are provided by complements of pseudoconvex domains in $C^{n}$.

Local maximum sets in the spectrum of a uniform algebra were studied by Wermer [15]. In this context the simplest local maximum sets are analytic discs.

If $F=$ the class of all $k$-plurisubharmonic functions on $C^{n}$ (where $0 \leq k \leq n-2)$, then $F$-maximum sets are identical with $k$-maximum sets studied in $[9, \S 2]$. Many results given below generalize those of [9].

We give next a local characterization of $F$-maximum sets in which axiom (1.8) is assumed. However, in most cases this local characterization is not used, and so we assume (1.8) only when it is necessary.

Proposition 4.2. Let $F$ be a class of usc functions on a locally compact space $M$. Assume that (1.8) holds. Let $V \subset M$ be open and $X=\bar{X} \cap V$ and let, for every $x,\left\{B_{t}(x)\right\}_{t \in T}$ be a fixed basis of relatively compact neighborhoods of $x$. Then the following conditions are 
equivalent:

(a) $X$ is an $F$-maximum set,

(b) for every $x \in X$ and for every $B_{t}(x)$, such that $\bar{B}_{t}(x) \subset V$, and for every $f \in F\left(\right.$ nbhd $\left.\bar{B}_{t}(x)\right)$

$$
f(x) \leq \max f \mid\left(X \cap \partial B_{t}(x)\right),
$$

(c) there does not exist $x \in X$, a neighborhood $B_{t}(x)$ and $f \in$ $F\left(B_{t}(x)\right)$, such that

$$
f(x)=0>f(y), \quad y \in X \cap B_{t}(x) \backslash\{x\} .
$$

Proof. Implications (a) $\Rightarrow$ (b) $\Rightarrow$ (c) are obvious. If (a) fails, there is a compact $K \subset M$ and a function $f_{1} \in F($ nbhd $K)$, such that $\max f_{1}|K \cap X>\max f|(\partial K) \cap X$. Applying Proposition 1.6 to $K_{1}=$ $K \cap X$ and $L_{1}=(\partial K) \cap X$, we get $x \in K_{1} \backslash L_{1}$ and $v \in A F\left(\right.$ nbhd $\left.K_{1}\right)$, such that $\left(f_{1}+v\right)(x)=0>\left(f_{1}+v\right)(y)$ for $y \neq x, y \in K_{1}$. Letting $f=\left(f_{1}+v\right) \in F$ and choosing $B_{t}(x)$ small enough, we obtain contradiction with (c). Thus, (c) $\Rightarrow($ a).

REMARK. By the last proposition, if $F$ satisfies (1.8), then $X$ is an $F$ maximum set, if and only if the class $F \mid X$ (consisting of restrictions of functions in $F$ to $X$ ) has local maximum property (1.11) on $X$. (Note that we do not assume the local maximum property of $F$ on M.)

The next corollary follows directly from Definition 4.1 and the last proposition (part (c)).

Corollary 4.3. Let $F$ be a class of usc functions on $M$ and let $X \subset M$. Then

(i) if $X$ is an $F$-maximum set and $U$ is open in $M$, then $X \cap U$ is an $F$-maximum set,

(ii) if $F$ satisfies axiom (1.8) and $\left\{U_{t}\right\}_{t \in T}$ is an open covering of $X$, such that $U_{t} \cap X$ is an $F$-maximum set for every $t \in T$, then $X$ is an $F$-maximum set.

COROLlary 4.4. Let $F$ be a class of usc functions on $M$. Let $K, Z$ be compact sets and $u \in \operatorname{usc}(Z)$, where $K \subset Z \subset M$. Assume that $Z \backslash K$ is an $F$-maximum set and $u \mid Z \backslash K \in F(Z \backslash K)$. Then $\max u|Z \leq \max u| K$.

Proof. Suppose, to the contrary, that $\max u|Z>\max u| K$. Then, there is an open relatively compact neighborhood $V$ of $K$, such that 
$\max u|(Z \backslash V)>\max u| \bar{V}$. Choose a compact $N \subset M$ so that $N \cap K=$ $\varnothing$ and $Z \subset \operatorname{Int}(N) \cup V$. Then, $Z \backslash V \subset N,(\partial N) \cap(Z \backslash K) \subset V$ and $(Z \backslash K) \cap N$ is compact. Hence, $\max u|N \cap(Z \backslash K)>\max u|(\partial N) \cap$ $(Z \backslash K)$.

Since $u \in F(Z \backslash K)$, there exist $u_{n} \in F($ nbhd $N), n=1,2, \ldots$, such that $u_{n}(x) \searrow u(x), x \in N$. If $n$ is large enough, we obtain, by the last inequality, that $\max u_{n}\left|N \cap(Z \backslash K)>\max u_{n}\right|(\partial N) \cap(Z \backslash K)$, which contradicts the assumptions.

REMARK 4.5. If localization axiom (1.8) is assumed, the set $Z \backslash V$ in the above proof can be made a singleton and then the proof applies to localized completions of the class $F$ and their transfinite iterates, in the spirit of Rickart [7].

Let $X$ be a locally compact subset of $M$. Then $X=\bar{X} \cap V$, where $V$ is open in $M$. We will call the function

$$
\chi_{X}(x)=\chi_{X}^{V}(x)= \begin{cases}0, & x \in X, \\ -\infty, & x \in V \backslash X,\end{cases}
$$

a "characteristic function" of $X$ relative to $V$.

Proposition 4.6. Let $F$ be a class of usc functions on $M$. Let $X$, $V \subset M$, where $V$ is open and $X=\bar{X} \cap V$. Assume that $F$ satisfies axiom (1.8). Then $X$ is an F-maximum set, if and only if the characteristic function $\chi_{X}=\chi_{X}^{V}$ is of class $F^{\mathrm{d}}(V)$.

Proof. Follows immediately from Lemma 2.8, Proposition 4.2 and the fact that $\left(\chi_{X}+f\right) \mid V \backslash X \equiv-\infty$, for every $f$. (Cf. [9, Theorem 2.5] for a similar argument.)

Corollary 4.7. Let $X \subset M$ and $F, F_{1}$ be two classes of usc functions on $M$, both satisfying axiom (1.8). Assume that $F^{\mathrm{d}}=F_{1}^{\mathrm{d}}$. Then $X$ is an $F$-maximum set, if and only if it is an $F_{1}$-maximum set. In particular, $F$ - and $F^{\mathrm{dd}}$-maximum sets are the same.

Proof. The first statement is a direct consequence of Proposition 4.6. The second statement is a special case with $F_{1}=F^{\mathrm{dd}}$, since $F^{\text {ddd }}=F^{\text {d }}$, by Proposition 2.4 .

REMARK 4.8. Clearly, $F^{\text {dd }}$ is usually a much larger class than $F$. For example, when $F=$ the class of all pluriharmonic quadratic polynomials on $\mathbf{C}^{n}$, then $F^{\mathrm{dd}}=$ the class of all plurisubharmonic functions on $\mathbf{C}^{n}$. (Follows from the results of [11].) 
Proposition 4.9. Let $F$ be a class of usc functions on $M$ satisfying axiom (1.8). Let $\left\{X_{t}\right\}_{t \in T}$ be a family of $F$-maximum sets, such that their union $X=\bigcup_{t \in T} X_{t}$ is locally compact. Then $X$ is an $F$-maximum set.

Proof. If not, then, by Proposition 4.2 (c), there are $x \in X$, a neighborhood $B$ of $x$ and $f \in F(B)$, such that $f(x)=0>f(y)$ for $y \in X \cap B \backslash\{x\}$. If $x \in X_{t}$, then $f(x)=0>f(y)$ for $y \in X_{t} \cap B \backslash\{x\}$, which contradicts the assumption that $X_{t}$ is an $F$-maximum set.

Proposition 4.10. Let $F$ be a class of usc functions on $M$. Assume that $F$ either satisfies axiom (1.9)(i) or consists of continuous functions only. Then

(i) whenever $Y \subset M$ is locally compact and $X_{t}, t \in T$, are $F$ maximum sets, such that $X_{t}=\bar{X}_{t} \cap Y$, then $X^{*}$ is an F-maximum set, where $X=\bigcup_{t \in T} X_{t}$ and $X^{*}=\bar{X} \cap Y$,

(ii) if $Y \subset M$ is locally compact and $X_{s}=\bar{X}_{s} \cap Y, s \in S$, form a net of F-maximum sets, then $X_{\infty}=\operatorname{LimSup}_{s} X_{s}$ is an F-maximum set, provided it is non-empty.

(iii) let $\left\{K_{s}\right\}_{s \in S}$ and $\left\{Z_{s}\right\}_{s \in S}$ be nets of compact subsets, such that both $\operatorname{Lim} \operatorname{Sup}_{s} Z_{s}=Z_{\infty}$ and $\operatorname{Lim} \operatorname{Sup}_{s} K_{s}=K_{\infty}$ exist and are compact sets. Assume that $Z_{s} \backslash K_{s}, s \in S$, are $F$-maximum sets. Then $Z_{\infty} \backslash K_{\infty}$ is an F-maximum set.

Proof. (i) Suppose $X^{*}$ is not an $F$-maximum set, i.e. there exist: $x^{*} \in X^{*}, K$ compact with $K \cap X^{*}$ compact and $f \in F\left(\operatorname{nbhd}\left(K \cap X^{*}\right)\right)$, such that $x^{*} \in K \backslash \partial K$ and $f\left(x^{*}\right)>\max f \mid(\partial K) \cap X^{*}$.

We consider first the case when axiom (1.9)(i) holds. Choose a relatively compact neighborhood $W$ of $(\partial K) \cap X_{\infty}$, so that $f \in$ $F\left(\operatorname{nbhd}\left(\bar{W} \cup K \cap X_{\infty}\right)\right)$ and $f\left(x_{0}\right)>\max f \mid \bar{W}$. Choose a compact set $K_{1}$, so that $K_{1} \cap X_{\infty}$ is compact, $K \cap X_{\infty} \cup \bar{W} \subset \operatorname{Int}\left(K_{1}\right)$, and $f \in F\left(\right.$ nbhd $\left.K_{1}\right)$. Choose $\varepsilon>0$ and $\varphi \in C\left(K_{1}\right)$, so that $\varphi>f$ on $K_{1}$ and

$$
f\left(x_{0}\right)-\varepsilon>\max \varphi \mid \bar{W} .
$$

We apply now axiom (1.9)(i) to data $x^{*}, L_{1}=K \cap X_{\infty}, K_{1}$ and $\varphi$. Let $V$ be a neighborhood of $x^{*}$ with properties postulated by (1.9)(i) and choose $s \in S$ and $x_{s} \in X_{s}$, so that $x_{s} \in V$. By (1.9)(i), there is 
$f_{s}^{\prime} \in F\left(\right.$ nbhd $\left.L_{1} \cup V\right)$, i.e. $f_{s}^{\prime} \in F\left(\operatorname{nbhd}\left(K \cap X_{\infty}\right)\right)$, such that $f_{s}^{\prime}(x)>$ $f\left(x_{0}\right)-\varepsilon$ and $f_{s}^{\prime}<\varphi$ on $L_{1} \cup \bar{V}$. Combining these inequalities and (4.1), we get

$$
f_{s}^{\prime}\left(x_{s}\right)>\max \varphi|\bar{W} \geq \max \varphi|(\partial K) \cap X_{s} \geq \max f_{s}^{\prime} \mid(\partial K) \cap X_{s},
$$

which is impossible, since $X_{s}$ is an $F$-maximum set.

In case $F$ consists of continuous functions only, most of the above argument can be omitted and we can simply take $f_{s}=f$.

(ii) By the definition $X_{\infty}=\bigcap_{\sigma} X^{\sigma}$, where $X^{\sigma}=Y \cap \mathrm{Cl}\left(\bigcup_{s \geq \sigma} X_{s}\right)$. By (i), each $X^{\sigma}$ is an $F$-maximum set, relatively closed in $Y$. Moreover, $X^{\sigma}$ form a decreasing family, and so (ii) follows immediately from the next assertion.

Assertion 1. If $X_{s}, s \in S$, form a decreasing family of $F$-maximum sets, where $S$ is a directed partially ordered set, and $X_{s}=\bar{X}_{s} \cap Y$, where $Y$ is locally compact, then $X_{\infty}=\bigcap_{S} X_{s}$ is an $F$-maximum set.

Indeed, if $K$ is compact, $K \cap X_{\infty}$ is compact, and $f \in$ $F\left(\right.$ nbhd $\left.K \cap X_{\infty}\right)$, then there is $s_{0} \in S$, such that $f \in F\left(\right.$ nbhd $K \cap X_{s}$ ) for $s \geq s_{0}$. Then

$$
\begin{aligned}
\max f \mid K \cap X_{\infty} & =\lim _{s} \max f\left|K \cap X_{s} \leq \lim _{s} \max f\right|(\partial K) \cap X_{s} \\
& =\max f \mid(\partial K) \cap X_{\infty} .
\end{aligned}
$$

(iii) The following assertion is an easy consequence of Definition 4.1.

Assertion 2. A locally compact set $X \subset M$ is an $F$-maximum set, if and only if for every open set $W$, such that $\bar{W} \cap X$ is compact, the intersection $W \cap X$ is an $F$-maximum set.

In the setting of (iii), if $\bar{W} \cap\left(Z_{\infty} \backslash K_{\infty}\right)$ is compact, we can assume without loss of generality that $\bar{W} \cap K_{\infty}=\varnothing$. Since $\operatorname{Lim} \operatorname{Sup}_{s} K_{s}=K_{\infty}$, there is $s_{0} \in S$, such that $K_{s} \subset M \backslash \bar{W}$ for $s \geq s_{0}$. Then, the sets $Y:=$ $W, X_{s}=Z_{s} \cap W=\left(Z_{s} \backslash K_{s}\right) \cap W$ and $X_{\infty}=Z_{\infty} \cap W=\left(Z_{\infty} \backslash K_{\infty}\right) \cap W$ satisfy assumptions of part (ii) (for $s \geq s_{0}$ ), and so the latter is an $F$-maximum set for every $W$ under consideration. By Assertion 2, $Z_{\infty} \backslash K_{\infty}$ is an $F$-maximum set.

Definition 4.11. Let $F$ be a class of usc functions on $M$, which satisfies axiom (1.9)(i) or consists of continuous functions only. Let $K \subset Y \subset M$, where $K$ is compact and $Y$ locally compact.

(a) We say that a set $Z \subset Y$ is a saturation of $K$ relative to $Y$, if $Z$ is compact and $Z \backslash K$ is an $F$-maximum set. 
(b) We call the union of all saturations of $K$ relative to $Y$ the saturation of $K$ relative to $Y$ and denote $\operatorname{Sat}_{Y}(K)$ or, more precisely, $\operatorname{Sat}_{Y}^{F}(K)$.

Note that the saturation of $K$ does not have to be compact, in general, and in such an instance it is not a saturation.

The saturation is an object similar to the polynomial hull. In fact, if $Y=M=C^{n}$, and $F=$ the class of all plurisubharmonic functions on $C^{n}$, then $\operatorname{Sat}_{Y}^{F}(K)=$ the polynomial hull of $K$. A more general result of this type will be proven in [14]. However, the identity of the saturation and of the hull (with respect to some class of functions, cf. Definition 4.17 below) holds only under strong assumptions about the class $F$ and the set $Z$. Note also that the saturation is built up by adding more and more to the set $K$, while the hull is obtained by removing some parts from the ambient space $M$. In view of these observations, the notion of the saturation seems to merit a study, independently on that of the hull.

COROLlary 4.12. Let $F, M, K, Y$ satisfy assumptions of Definition 4.11. Then

(i) $\operatorname{Sat}_{Y}(K)$ is relatively closed in $Y$ provided it is relatively compact in $Y$.

(ii) $\operatorname{Sat}_{Y}(K)$ is compact, if and only if it is relatively compact in $Y$. If $\operatorname{Sat}_{Y}(K)$ is compact, it is a saturation, i.e. $\operatorname{Sat}_{Y}(K) \backslash K$ is an $F$-maximum set.

Proof. (i) Assume that $\operatorname{Sat}_{Y}(K) \subset Y_{0} \subset Y$, where $Y_{0}$ is compact. Let $x \in \mathrm{Cl}\left(\operatorname{Sat}_{Y}(K)\right), x \notin K$. Then there exists a net $\left(x_{t}\right)_{t \in T}, T$ being an ordered set, such that $\lim _{t} x_{t}=x$ and $x_{t} \in Z_{t}$, where $Z_{t}$ is a saturation of $K$, for $t \in T$. Since all the $Z_{t}$ are subsets of the compact set $Y_{0}$, $\operatorname{Lim} \operatorname{Sup}_{s} Z_{t}=Z_{\infty}$ exists and is a compact set. By Proposition 4.10 (iii), $Z_{\infty} \backslash K$ is an $F$-maximum set and so $Z_{\infty}$ is a saturation of $K$. Hence, $x \in \operatorname{Sat}_{Y}(K)$, seeing that $x \in Z_{\infty}$. This proves (i).

(ii) If $\operatorname{Sat}_{Y}(K)$ is compact, $\operatorname{Sat}_{Y}(K) \backslash K$ is relatively closed in $Y_{1}=$ $Y \backslash K$ and is the union of $F$-maximum sets $Z_{t} \backslash K$ (where $Z_{t}$ is any saturation), which are all relatively closed in $Y_{1}$. By Proposition 4.10 (i), $\operatorname{Sat}_{Y}(K) \backslash K$ is an $F$-maximum set.

If $\operatorname{Sat}_{Y}(K)$ is relatively compact in $Y$, it is relatively closed, by (i), and so is compact.

COROLlary 4.13. Let $F$ be a class of usc functions on $M$ satisfying the localization axiom (1.8). Assume that $\operatorname{Sat}_{Y}(K)$ is compact, 
where $K$ is compact, $Y$ locally compact and $K \subset Y \subset M$. Then, $\operatorname{Sat}_{Y}\left(\operatorname{Sat}_{Y}(K)\right)=\operatorname{Sat}_{Y}(K)$.

Proof. Let $Z=\operatorname{Sat}_{Y}(K)$ and suppose $\operatorname{Sat}_{Y}(Z) \neq Z$. Then, there is $Z_{1} \subset Y$, such that $Z_{1}$ is compact and $Z_{1} \backslash Z$ is an $F$-maximum set. Let $Z_{2}=Z_{1} \cup Z$. Then $Z_{2}$ is compact and $Z_{2} \backslash K$ is an $F$-maximum set, by Proposition 4.9, because it is locally compact and is the union of two $F$-maximum sets $Z_{1} \backslash Z$ and $Z \backslash K$. Thus, $Z_{2}$ is a saturation of $K$, and so $Z_{2} \subset Z$.

REMARK 4.14. It follows immediately from Corollary 4.7 that, if $F$ and $F_{1}$ are two classes of usc functions on $M$, both satisfying condition (1.8), and such that $F^{\mathrm{d}}=F_{1}^{\mathrm{d}}$, then $\operatorname{Sat}_{Y}^{F}(K)=\operatorname{Sat}_{Y}^{F}(K)$.

LEMMA 4.15. Let $F$ be a class of usc functions on $M$ satisfying axioms (1.5) and (1.8). Let $u: U \rightarrow R$ be an usc function, where $U$ is open in $M$. Let $X_{t}=\{x \in U: u(x) \geq t\}$. Then, the following conditions are equivalent:

(i) all the $X_{t}$ 's, $t \in R$, are $F$-maximum sets,

(ii) for every continuous, non-decreasing function $\varphi: R \rightarrow R$, the composition $x \rightarrow \varphi(u(x))$ is of $F^{\mathrm{d}}$-class,

(iii) the same as (ii), with $\varphi C^{(2)}$-smooth and $\varphi^{\prime}(x)>0$ on $R$.

A natural application of this lemma is the next corollary, in which presence of an additional structure implies that one of the sets $X_{t}$ is an $F$-maximum set, if and only if all of them are. The corollary will be used in [13].

Corollary 4.16. Let $M$ be a locally compact space and $\tilde{M}=M \times$ $R^{N}$. Let $F$ be a class of usc functions on $\tilde{M}$, satisfying axioms (1.5) and (1.8), and invariant with respect to homoteties $(x, y) \rightarrow(x, c y)$, $(x, y) \in M \times R^{N}, c>0$. Let $G \subset M$ be open and $p: G \times R^{N} \rightarrow[0, \infty)$ be a lower semi-continuous function, such that $p(x, r y)=r p(x, y)$, $r \geq 0$, and $p(x, y)=0$, if and only if $y=0$. Denote

$$
X=\left\{(x, y) \in G \times\left(R^{N} \backslash\{0\}\right): p(x, y) \leq 1\right\} .
$$

Then

(a) $X$ is an $F$-maximum set, if and only if for every $C^{(2)}$-smooth function $\varphi: R \rightarrow R$, such that $\varphi^{\prime}(x)>0, x \in R$, the composition $(x, y) \rightarrow \varphi(-\log p(x, y))$ belongs to $F^{\mathrm{d}}\left(G \times\left(R^{N} \backslash\{0\}\right)\right)$, 
(b) if, in addition, $F$ satisfies axiom (1.12), then $X$ is an $F$-maximum set, if and only if the function $(x, y) \rightarrow \min (-\log p(x, y), 0)$ is of class $F^{\mathrm{d}}\left(G \times\left(R^{N} \backslash\{0\}\right)\right)$.

Proof of Lemma 4.15. Obviously, (ii) $\Rightarrow$ (iii). Conversely, (iii) $\Rightarrow$ (ii). Indeed, if $\varphi: R \rightarrow R$ is continuous and non-decreasing, there exist functions $\varphi_{n} \in C^{(2)}(R)$, such that $\varphi_{n}^{\prime}(t)>0, n=1,2, \ldots$, and $\varphi_{n}(t) \searrow \varphi(t)$, for $t \in R$. Then, $\varphi_{n} \circ u \in F^{\mathrm{d}}$ and $\varphi_{n}(u(x)) \searrow \varphi(u(x))$, $x \in U$, and so $\varphi \circ u \in F^{\mathrm{d}}(U)$, by Lemma 2.9.

(i) $\Rightarrow$ (ii). Denote $\chi_{t}(x)=\chi_{X_{t}}^{U}(x)$. By Proposition 4.6, $x_{t} \in F^{\mathrm{d}}(U)$. Let $h_{t}(x)=\varphi(t)+\chi_{t}(x)$. Since $F^{\mathrm{d}}+$ const $\subset F^{\mathrm{d}}$, by Lemma 2.9, $h_{t} \in F^{\mathrm{d}}(U)$, for all $t \in R$. Since $h_{t} \equiv-\infty$ on $U \backslash X_{t}$ and $\varphi$ is nondecreasing, $h_{t} \leq \varphi \circ u$, for $t \in R$. On the other hand, if $x^{*} \in U$ and $t^{*}=u\left(x^{*}\right)$, then $h_{t^{*}}\left(x^{*}\right)=\varphi\left(t^{*}\right)+\chi_{t^{*}}\left(x^{*}\right)=\varphi\left(u\left(x^{*}\right)\right)$. Thus, $(\varphi \circ u)(x)=\sup _{t} h_{t}(x), x \in U$. Since $\varphi \circ u$ is usc, $\varphi \circ u \in F^{\mathrm{d}}(U)$, by Lemma 2.9.

(ii) $\Rightarrow$ (i). Fix $t \in R$ and let $\varphi(y)=-\infty$ for $y<t$ and 0 for $y \geq t$. Further, let $\varphi_{n}(y)=n(y-t)$, for $y<t$ and 0 for $y \geq t, n=1,2, \ldots$. By (ii), $\varphi_{n} \circ u \in F^{\mathrm{d}}(U)$. Since $\varphi_{n} \circ u(x) \searrow \varphi \circ u(x)=\chi_{t}(x), \chi_{t} \in F^{\mathrm{d}}(U)$ by Lemma 2.9. Thus, $X_{t}$ is an $F$-maximum set, by Proposition 4.6.

Proof of Corollary 4.16. (a) Let $U=G \times\left(R^{N} \backslash\{0\}\right)$ and $u(x, y)=$ $-\log p(x, y)$. If $X_{t}, t \in R$, are defined as in the last proof, then $X=$ $X_{0}$, and every $X_{t}$ is the image of $X$ under the map $(x, y) \rightarrow\left(x, e^{t} y\right)$. Thus, $X$ is an $F$-maximum set, if and only if all the $X_{t}$ 's are, and so Lemma 4.15 implies (a).

(b) In the same way, the necessity in (b) follows from Lemma 4.15(ii). As for sufficiency, if $v \in F^{\mathrm{d}}(U), U=G \times\left(R^{N} \backslash\{0\}\right)$, where $v(x, y)=\min (-\log p(x, y), 0)$, then $n v \in F^{\mathrm{d}}(U), n=1,2, \ldots$, by Proposition 1.14(i). Since $n v(x, y) \searrow \chi_{X}^{U}(x, y), \chi_{X} \in F^{\mathrm{d}}(U)$, by Lemma 2.9, and so $X$ is an $F$-maximum set.

DEFINITION 4.17 (Krantz [5, p. 106]). Let $F(Y)$ be a family of functions defined on a set $Y$ and $K \subset Y$. We say that $K$ is $F(Y)$ convex, or convex with respect to $F(Y)$, if for every $y \in Y \backslash K$ there is $g \in F(Y)$, such that $\sup g \mid K<g(y)$. 
The next corollary will be applied in [13]. A related result, for analytic multifunctions in one-dimensional setting, was obtained by Ransford [6, Theorem 5.1].

CoRollary 4.18. Let $F$ be a class of usc functions on $\tilde{M}=M \times R^{N}$, satisfying assumptions of Definition 4.11, and $G \subset M$ be open and relatively compact in $M$. Let $\zeta \rightarrow K(\zeta): \partial G \rightarrow 2^{R^{N}}$ be an usc, compactvalued function. Assume that for each $b \in \partial G$, the set $\{b\} \times K(b)$ is $A F\left(\bar{G} \times R^{N}\right)$-convex and there are barrier functions $u_{n}^{b} \in F\left(\bar{G} \times R^{N}\right)$, $n=1,2, \ldots$, such that

$$
\begin{aligned}
u_{n}^{b} \mid\{b\} \times R^{N}=0 ; \quad 0 & \geq u_{n}^{b}(z, w) \searrow-\infty, \\
& (z, w) \in(\bar{G} \backslash\{b\}) \times R^{N} .
\end{aligned}
$$

Then, Sat ${\bar{G} \times R^{N}}(\operatorname{gr}(K)) \cap(\partial G) \times R^{N}=\operatorname{gr}(K)(=$ the graph of $K(\cdot))$.

Proof. Recall that $\operatorname{gr}(K)=\left\{(z, w) \in \partial G \times R^{N}: w \in K(z)\right\}$. We have to show that, if $Z$ is a compact subset of $\bar{G} \times R^{N}$, such that $Z \backslash \operatorname{gr}(K)$ is an $F$-maximum set, then $Z_{b} \subset K(b)$, where

$$
Z_{b}=\left\{w \in R^{N}:(b, w) \in Z\right\},
$$

cf. Definition 4.11(a). Suppose, on the contrary, that there is $w_{0} \in$ $Z_{b} \backslash K(b)$. By the $A F$-convexity of $\{b\} \times K(b)$, there is $g \in$ $A F\left(\bar{G} \times R^{N}\right)$, such that $g\left(b, w_{0}\right)>2 \varepsilon+\max g \mid\{b\} \times K(b)$, for some $\varepsilon>0$. Let $v_{n}(z, w)=g(z, w)+u_{n}^{b}(z, w),(z, w) \in \bar{G} \times R^{N}$. By Definition 1.3 and Notation 1.1, $v_{n} \in(A F)+F \subset F\left(\bar{G} \times R^{N}\right)$. Seeing that $v_{n}(b, w)=g(w), w \in R^{N}, v_{n}(z, w) \searrow-\infty$, for $(z, w) \in Z \backslash\{b\} \times R^{N}$, and taking into account that $Z$ is compact and $v_{n}$ are usc, we conclude that for $n$ large enough,

$\max v_{n}\left|Z \geq g\left(b, w_{0}\right)>\varepsilon+\max g\right|\{b\} \times K(b)>\max v_{n} \mid \operatorname{gr}(K)$.

This contradicts Corollary 4.4 .

COROllary 4.19. Let $M, \tilde{M}, F$ be as in the last corollary. Let $G \subset$ $M$ be an open and relatively compact subset of $M$ and $b \in \partial G$ admit a sequence of barrier functions $\left(u_{n}^{b}\right) \subset F\left(\bar{G} \times R^{N}\right)$ satisfying condition (4.2). Let $Z \subset \bar{G} \times R^{N}$ be an $F$-maximum set. Then $X=Z \cap\{b\} \times R^{N}$ is an AF-maximum set.

Proof. Suppose not, then there exist a compact subset $N \subset \tilde{M}$ and $g \in A F(\operatorname{nbhd}(N \cap X))$, such that $X \cap N$ is compact and

$$
\max g|(\partial N) \cap X<\max g| N \cap X
$$


and (without loss of generality) sup $g<C_{0}<\infty$. Choose now a compact set $N_{0}$, such that $N_{0} \subset N \cap \operatorname{Dom}(g), N \cap Z$ is compact, $N_{0} \cap X=N \cap X$ and $\partial N_{0} \cap X=\partial N \cap X$. Then $Z \cap N_{0} \cap\{b\} \times R^{N}=X \cap N$ and $Z \cap N_{0} \subset \bar{G} \times R^{N}$. Using this, inequality (4.4) and the property that $0>u_{n}^{b}(z, w) \searrow-\infty$ on $(\bar{G} \backslash\{b\}) \times R^{N}$, we conclude that for $n$ large enough $\max \left(g+u_{n}^{b}\right)\left|\partial N_{0} \cap Z<\max g\right| N_{0} \cap X$. Since $u_{n}^{b}(b, w)=0$, $w \in R^{N}$ and $X \subset Z$, we get

$$
\max \left(g+u_{n}^{b}\right)\left|\partial N_{0} \cap Z<\max \left(g+u_{n}^{b}\right)\right| N_{0} \cap Z
$$

By the definition of $F\left(\bar{G} \times R^{N}\right)$, cf. Notation 1.1, we can approximate each $u_{n}^{b} \mid N_{0} \cap\left(\bar{G} \times R^{N}\right)$, in a decreasing fashion, by functions $v \in$ $F\left(\operatorname{nbhd}\left(N_{0} \cap \bar{G} \times R^{N}\right)\right)$. Replacing $u_{n}^{b}$ in (4.5) by a sufficiently good approximation $v$, we get $\max (g+v)\left|\partial N_{0} \cap Z<\max (g+v)\right| N_{0} \cap Z$. Since $g+v \in A F+F \subset F$, and $Z$ is an $F$-maximum set, we get a contradiction.

5. Absolutely pseudoconvex and relatively pseudoconvex sets. Perhaps the most natural motivation of the notion of $P$-pseudoconvex sets comes from the study of convex hulls relative to a class of functions. This approach corresponds to holomorphic convexity in the classical case and we will follow it in [14]. Already at this stage, however, one can define $P$-pseudoconvexity by a version of kontinuitatsatz, with $P$-maximum sets replacing the usual analytic discs.

Permanent Assumption. We will assume throughout the whole section that $F$ is a class of usc functions on the given locally compact space $M$, satisfying conditions of Definition 4.11 .

Definition 5.1. (i) Let $Y \subset M$. We say that $Y$ is $F$-pseudoconvex, if $Y$ is locally compact and if, whenever two nets $\left(Z_{t}\right)_{t \in T},\left(X_{t}\right)_{t \in T}$ are such that

$$
\begin{aligned}
& Z_{t}, X_{t} \subset Y, \quad Z_{t}, X_{t} \text { are compact, } \quad t \in T, \\
& Z_{t} \backslash X_{t} \text { is an } F \text {-maximum set, } \quad t \in T,
\end{aligned}
$$

(5.3) $\operatorname{Lim} \operatorname{Sup}_{t} X_{t}=X_{\infty}$ exists and is a non-empty compact set, then the limit $Z_{\infty}=\operatorname{Lim} \operatorname{Sup}_{t} Z_{t}$ exists and is a compact set.

(ii) Let $Y \subset R \subset M$. We say that $Y$ is relatively $F$-pseudoconvex in $R$, if $Y$ and $R$ are locally compact, and for every two nets $\left(Z_{t}\right)_{t \in T}$, $\left(X_{t}\right)_{t \in T}$ of subsets of $Y$, satisfying conditions (5.1) through (5.3) and 
such that $\operatorname{Lim} \operatorname{Sup}_{t} Z_{t}=Z_{\infty}$, considered relative to $R$, exists and is compact, the set $Z_{\infty}$ must be contained in $Y$.

REMARK. In the above definition, $\operatorname{Lim} \operatorname{Sup}_{t} Z_{t}$ is understood in a more restrictive sense than in Proposition 4.10, Proof of (ii). Namely, we require also that for every neighborhood $U$ of $Z_{\infty}$, there is $t_{0} \in T$, such that $Z_{t} \subset U$ for $t \geq t_{0}$.

Proposition 5.2. Let $Y \subset R \subset M$ be locally compact sets. Then

(a) if $Y$ is $F$-pseudoconvex, then $Y$ is relatively $F$-pseudoconvex in $R$,

(b) if $R$ is $F$-pseudoconvex and $Y$ is relatively $F$-pseudoconvex in $R$, then $Y$ is $F$-pseudoconvex,

(c) if $Y$ is relatively compact in $R$ and relatively $F$-pseudoconvex in $R$, then $Y$ is $F$-pseudoconvex,

(d) if $R$ is compact and $Y$ is relatively $F$-pseudoconvex in $R$, then $Y$ is $F$-pseudoconvex,

(e) if $u \in F(R)$ and $R_{C}:=\{x \in R: u(x)<C\}$, where $-\infty<C<$ $+\infty$, then $R_{C}$ is relatively $F$-pseudoconvex in $R$. In particular, if $R$ is $F$-pseudoconvex, then so is $R_{C}$.

Proof (Sketch). (a) and (b) are obvious, and (d) follows directly from (c).

(c) Since $\bar{Y}$ is compact and $\bar{Y} \subset R, Z_{\infty}=\operatorname{Lim~Sup}_{t} Z_{t}$ exists, relative to $\bar{Y}$. It is then easy to observe that $\operatorname{Lim} \operatorname{Sup}_{t} Z_{t}$, relative to $R$, exists and is equal to $Z_{\infty}$. Since $Y$ is relatively pseudoconvex in $R, Z_{\infty} \subset Y$. Now, it suffices to observe that $\operatorname{Lim} \operatorname{Sup}_{t} Z_{t}$, relative to $Y$, exists as well (and is equal to $Z_{\infty}$ ). We omit further details.

(e) Let $Z_{t}, X_{t}, T$ satisfy conditions of Definition 5.1(ii), with $Y=$ $R_{C}$. We have to show that $Z_{\infty} \subset R_{C}$. Since $X_{\infty} \subset R_{C}, u \mid X_{\infty}<C$. By Proposition 4.10(ii), $Z_{\infty} \backslash X_{\infty}$ is an $F$-maximum set and, by Corollary 4.4, $u\left|Z_{\infty} \leq \max u\right| X_{\infty}<C$. Thus, $Z_{\infty} \subset R_{C}$.

REMARK. In contrast with the standard usage, we consider also non-open pseudoconvex sets. This allows for more uniformity in exposition and will be also useful in [13]. We note that every compact set is $F$-pseudoconvex.

Proposition 5.3. Let $Y, R, S \subset M$ be locally compact.

(a) If $Y \subset R$ and $Y$ is relatively $F$-pseudoconvex in $R$, then $Y \cap S$ is relatively $F$-pseudoconvex in $R \cap S$. 
(b) If $Y$ is relatively $F$-pseudoconvex in $R$ and $R$ is relatively $F$ pseudoconvex in $S$, then $Y$ is relatively $F$-pseudoconvex in $S$.

Proof (Sketch). Similarly as in the proof of (b) given next, (a) follows directly from Definition 5.1(ii).

(b) Let $\left(Z_{t}\right),\left(X_{t}\right)$ be nets of compact subsets of $Y$ (satisfying conditions of Definition 5.1(ii)) for testing of the relative $F$-pseudoconvexity of $Y$ in $S$. In particular, $\operatorname{Lim} \operatorname{Sup}_{t} Z_{t}=Z_{\infty}$, relative to $S$, exists and $Z_{\infty}$ is a compact subset of $S$. Since $R$ is relatively $F$-pseudoconvex in $S, Z_{\infty} \subset R$. Clearly, $Z_{\infty}=\operatorname{LimSup}_{t} Z_{t}$, relative to $R$. Since $Y$ is relatively $F$-pseudoconvex in $R, Z_{\infty} \subset Y$.

The proof of the next proposition is similar to the last one and is omitted.

Proposition 5.4. Let $Y_{i}, i \in I$, and $R$ be locally compact subsets of $M$. If every $Y_{i}, i \in I$, is relatively $F$-pseudoconvex in $R$, and the intersection $Y=\bigcap_{i \in I} Y_{i}$ is locally compact, then $Y$ is relatively $F$ pseudoconvex in $R$.

After these rudimentary observations on the general case (which will be continued in [14]), we will focus now on the case of $Y$ and $F$ of the special form, considered already in Corollary 4.16 (and relevant for the applications in [13]).

LEMMA 5.5. Let $M$ be a locally compact space and $\tilde{M}=M \times R^{N}$. Let $F$ be a class of usc functions on $\tilde{M}$ satisfying assumptions of Definition 4.11 and invariant with respect to maps $(x, y) \rightarrow(x, t y), t>0$. Let $G \subset M$ be locally compact and $Y \subset G \times M$ relatively open. Assume that sections $Y_{z}, z \in G$ (cf. (4.3)) form a locally uniformly bounded family of non-empty star-shaped subsets of $R^{N}$.Then $Y$ is $F$-pseudoconvex in $G \times R^{N}$, if and only if $\operatorname{Sat}_{G \times R^{N}}^{F}(K) \subset Y$, whenever $K \subset Y$ is compact.

REMARK. For classes $F$ with some additional properties, this result means that $Y$ is $F$-pseudoconvex, if and only if it is $F(Y)$-convex, cf. [14].

Proof. By Definition 4.11, an equivalent formulation is: if $K \subset Y$, $K$ and $Z$ are compact sets, and $Z \backslash K$ is an $F$-maximum set, then $Z \subset Y$. 
"Sufficiency" is now obvious: in the situation of Definition 5.1 (ii), $Z_{\infty} \subset Y$, because $X_{\infty} \subset Y$ and $Z_{\infty} \backslash X_{\infty}$ is an $F$-maximum set by Proposition 4.10(iii).

"Necessity". Suppose $K, Z$ are compact, $Z \backslash K$ is an $F$-maximum set and $Z \not \subset Y$, while $K \subset Y$. Let $Z^{t}, K^{t}$ denote the images of $Z, K$ under maps $(x, y) \rightarrow(x, t y), t>0$. Let $\tau$ be the smallest $t$, such that $Z_{t}$ intersects $\left(G \times R^{N}\right) \backslash Y$. Then $\tau \leq 1$, since $Z^{1}=Z$, and $K^{t} \subset Y$ for $t \leq \tau$, since $K^{1}=K \subset Y$ and $Y_{Z}$ are star-shaped. The nets $\left(Z^{t}\right)_{0<t<\tau},\left(K^{t}\right)_{0<t<\tau}$, with $Z_{\infty}=\operatorname{Lim} \operatorname{Sup}_{t \rightarrow \tau} Z^{t}=Z^{\tau}$ and $K_{\infty}=\operatorname{LimSup} \operatorname{Su}_{t \rightarrow \tau} K^{t}=K^{\tau}$ satisfy conditions of Definition 5.1(ii). Thus, $Z^{\tau} \subset Y$, which is a contradiction.

REMARK 5.6. The last lemma applies in particular when $G$ is a singleton, $G=\{\zeta\}$.

In the classical case, pseudoconvexity is a local property of the boundary. The next theorem is a partial result in this direction. (The general problem remains open.)

THEOREM 5.7. Let $M$ be a complex manifold on which globally holomorphic functions separate points, $\tilde{M}=M \times \mathbf{C}^{n}, F$ a class of functions on $\tilde{M}, G \subset M$ and $Y \subset G \times \mathbf{C}^{n}$. Assume that $F, Y$ satisfy all the assumptions of Lemma 5.5 and, in addition, $G$ is open, $Y_{z}, z \in G$, are circled, and $F$ is invariant with respect to the maps

$$
(z, w) \rightarrow(z, h(z) w),
$$

where $h(\cdot)$ is a nowhere-vanishing holomorphic function. Let $\left\{G_{t}\right\}_{t \in T}$ be an open covering of $G$. Then $Y$ is relatively $F$-pseudoconvex in $G \times \mathbf{C}^{n}$, if and only if $Y \cap\left(G_{t} \times \mathbf{C}^{n}\right)$ is relatively $F$-pseudoconvex in $G_{t} \times \mathbf{C}^{n}$ for every $t \in T$.

REMARK 5.8. Under the assumptions of the above theorem, if $Y$ is not relatively $F$-pseudoconvex in $G \times \mathbf{C}^{n}$, then there are compact sets $K, Z$ and point $a \in \pi(Z)$, where $\pi: M \times \mathbf{C}^{n} \rightarrow M$ is the standard projection, such that $K \subset Y, Z \backslash K$ is an $F$-maximum set, and $Z \subset$ $Y \cup\{a\} \times \mathbf{C}^{n}$, but $Z \not \subset Y$.

Proof of Remark 5.8. By Lemma 5.5, there are compact sets $K^{0}$, $Z^{0}$, such that $Z^{0} \subset G \times \mathbf{C}^{n}, K^{0} \subset Y, Z^{0} \not \subset Y$, and $Z^{0} \backslash K^{0}$ is an $F$-maximum set. Define

(5.5) $e^{u(z)}=\max \left\{t>0: t^{-1} Z_{z}^{0} \cap\left(\mathbf{C}^{n} \backslash Y_{z}\right) \neq \varnothing\right\}, \quad z \in \pi\left(Z^{0}\right)$,

(5.6) $e^{v(z)}=\max \left\{t>0:\left(t^{-1} K_{z}^{0}\right) \cap\left(\mathbf{C}^{n} \backslash Y_{z}\right) \neq \varnothing\right\}, \quad z \in \pi\left(K^{0}\right)$, 
where $K_{z}^{0}, Z_{z}^{0}, Y_{z}$ are defined by (4.3). Clearly, $u(z) \geq v(z), z \in$ $\pi\left(K^{0}\right)$ and one can show easily (details omitted) that $u(\cdot)$ and $v(\cdot)$ are usc functions on $\pi\left(Z^{0}\right)$ and $\pi\left(K^{0}\right)$ respectively. Since $K^{0} \subset Y$ and $Z^{0} \not \subset Y$,

$$
\max u>0>\max v .
$$

By Corollary A.3 and Proposition A.4, there is a holomorphic function $\rho: \operatorname{nbhd}\left(\pi\left(Z^{0}\right)\right) \rightarrow \mathbf{C}$ and $a \in \pi\left(Z^{0}\right)$, such that

$$
\begin{aligned}
& (u+\operatorname{Re} \rho)(a)=0>(u+\operatorname{Re} \rho)(z), \quad z \in \pi\left(Z^{0}\right) \backslash\{a\}, \\
& \max (v+\operatorname{Re} \rho) \mid \pi\left(K^{0}\right)<0 .
\end{aligned}
$$

Now let $h(z)=\exp \rho(z)$ and define compact sets $Z, K$ as the images of $Z^{0}, K^{0}$ under the map $(z, w) \rightarrow(z, h(z) w)$. Since $F$ is invariant with respect to such maps, $Z \backslash K$ is an $F$-maximum set. By (5.5) and (5.6),

$$
\begin{aligned}
& \max \left\{t>0: t^{-1} Z_{z} \cap\left(\mathbf{C}^{n} \backslash Y_{z}\right) \neq \varnothing\right\}=e^{u(z)+\operatorname{Re} \rho(z)}, \quad z \in \pi(Z), \\
& \max \left\{t>0: t^{-1} K_{z} \cap\left(\mathbf{C}^{n} \backslash Y_{z}\right) \neq \varnothing\right\}=e^{v(z)+\operatorname{Re} \rho(z)}, \quad z \in \pi(K),
\end{aligned}
$$

(we used here the fact that $Y_{z}$ are circled). These relations and (5.8), (5.9) imply $Z_{a} \cap\left(\mathbf{C}^{n} \backslash Y_{a}\right) \neq \varnothing, Z_{z} \subset Y_{z}$ for $z \in \pi(Z) \backslash\{a\}$, and $K_{z} \subset Y_{z}$ for $z \in \pi(K)$, i.e. $K \subset Y$.

Proof of Theorem 5.7. The condition is necessary by Proposition 5.3(a). Suppose it is not sufficient, then there are $K, Z, a$ with properties stated in Remark 5.8. Choose $G_{t}$, such that $a \in G_{t}$ and a relatively compact neighborhood $B$ of $a$ with $\bar{B} \subset G_{t}$. Let $Z_{1}=\left(\bar{B} \times \mathbf{C}^{n}\right) \cap Z$ and $K_{1}=\left(\bar{B} \times \mathbf{C}^{n}\right) \cap K \cup\left(\partial B \times \mathbf{C}^{n}\right) \cap Z$. Clearly, $K_{1}, Z_{1}$ are compact. Since $Z_{1} \backslash K_{1}=(Z \backslash K) \cap\left(B \times \mathbf{C}^{n}\right)$, it is an $F$-maximum set by Corollary 4.3(i). Moreover, by Remark 5.8, $K_{1} \subset Y \cap\left(G_{t} \times \mathbf{C}^{n}\right), Z_{1} \subset G_{t} \times \mathbf{C}^{n}$ and $Z_{1} \subset Y \cap\left(G_{t} \times \mathbf{C}^{n}\right) \cup\{a\} \times \mathbf{C}^{n}$, while $Z_{1} \not \subset Y \cap\left(G_{t} \times \mathbf{C}^{n}\right)$. By Lemma 5.5, this means that $Y \cap\left(G_{t} \times \mathbf{C}^{n}\right)$ is not relatively $F$-pseudoconvex in $G_{t} \times \mathbf{C}^{n}$.

We will consider now a property of a pair $Y \subset R$, which is, at least formally, stronger than the relative $F$-pseudoconvexity of $Y$ in $R$. Let $Y^{*}=Y \cup$ interior of $Y \cup(\bar{Y} \cap \partial R)$, relative to $\bar{R}$. By Proposition 5.3(a), relative $F$-pseudoconvexity of $Y^{*}$ in $\bar{R}$ implies that of $Y$ in $R$. The converse is open even in the classical case. (In case $\bar{Y}$ is compact in $R$, a positive answer would mean that relative $F$-pseudoconvexity of $Y$ in $R$ is equivalent to absolute $F$-pseudoconvexity of $Y^{*}$ ). We give a partial result in the context of Lemma 5.5. 
Corollary 5.9. Let $M, \tilde{M}, F, G, Y$ satisfy assumptions of Lemma 5.5. Assume additionally that $G, Y$ are relatively compact in $M$ and $\tilde{M}$ respectively, and for every $b \in \partial G$, there are "barrier" functions $u_{n}^{b} \in F\left(\bar{G} \times R^{N}\right)$ satisfying condition (4.2) of Corollary 4.18. Let $Y^{*}=$ interior of $Y \cup\left(\partial G \times R^{N}\right) \cap \bar{Y}$ relative to $\bar{G} \times R^{N}$. Assume that the fibers $\{b\} \times Y_{b}^{*}$ are $A F$-pseudoconvex. Then $Y=Y^{*} \cap\left(G \times R^{N}\right)$ is relatively $F$-pseudoconvex in $G \times R^{N}$, if and only if $Y^{*}$ is relatively $F$-pseudoconvex in $\bar{G} \times R^{N}$.

Proof. Only necessity requires proof. By Lemma 5.5, we have to show that, if $K, Z \subset \bar{G} \times R^{N}$ are compact sets, such that $K \subset Y^{*}$ and $Z \backslash K$ is an $F$-maximum set, then $Z \subset Y^{*}$. Let $b \in \partial G$; by Corollary 4.19, the set $\{b\} \times R^{N} \cap(Z \backslash K)=\{b\} \times Z_{b} \backslash\{b\} \times K_{b}$ is an $A F$-maximum set. Since the fiber $\{b\} \times Y_{b}^{*}$ is $A F$-pseudoconvex and contains $\{b\} \times K_{b}$, by Remark 5.6, $\{b\} \times Z_{b} \subset\{b\} \times Y_{b}$, for every $b \in \partial G$. By the compactness of $\bar{G}$ and $Z$, and relative openness of $Y$, there is an open neighborhood $V$ of $\partial G$, such that

$$
Z_{z} \subset Y_{z}, \quad z \in \bar{V} \cap \bar{G} .
$$

Let $N=G \backslash V, Z_{1}=Z \cap\left(N \times R^{N}\right)$ and $K_{1}=K \cap\left(N \times R^{N}\right) \cup Z \cap$ $\left(\partial N \times R^{N}\right)$. Then $N, K_{1}, Z_{1}$ are compact, $Z_{1} \subset G \times R^{N}$ and, by (5.10), $K_{1} \subset Y$. Furthermore, $Z_{1} \backslash K_{1}=($ Int $N) \times R^{N} \cap(Z \backslash K)$ is an $F$-maximum set. Since $Y$ is relatively $F$-pseudoconvex in $G \times R^{N}$, $Z_{1} \subset Y$ (by Lemma 5.5), which, together with (5.10), implies that $Z \subset Y^{*}$.

CoROllaRY 5.10. Let $M, F$ satisfy the assumptions of Lemma 5.5 and, in addition, axiom (1.8). Let $G \subset M$ be open and relatively compact and $z \rightarrow W(z): \bar{G} \rightarrow 2^{R^{N}}$ be a continuous family of compact strictly star-shaped (i.e. $t W(z) \subset$ Int $W(z), t \in(0,1), z \in \bar{G})$ neighborhoods of zero. Assume that $\operatorname{gr} W=\operatorname{Sat} \frac{F}{G} \times R^{N}(\operatorname{gr}(W \mid \partial G))$. Then the set $Y^{*}=\{(z, w): z \in \bar{G}, w \in \operatorname{Int} W(z)\}$, which is open in $\bar{G} \times R^{N}$, is relatively $F$-pseudoconvex in $\bar{G} \times R^{N}$.

Proof. By Lemma 5.5, we have to show that $\operatorname{Sat}_{\bar{G} \times R^{N}}(K) \subset Y^{*}$, whenever $K \subset Y^{*}$ is compact. By the continuity of $z \rightarrow W(z)$, there is $t \in(0,1)$, such that $K \subset \operatorname{gr}(t W)=\{(z, t w): z \in \bar{G}, w \in W(z)\}$. Clearly, the latter set is the saturation of $\{(b, t w): b \in \partial G, w \in W(b)\}$ (by the invariance property of $F$ ) and so is equal to its own saturation 
by Corollary 4.13. Thus, $\operatorname{Sat}_{\bar{G} \times R^{N}}(K) \subset \operatorname{gr}(t W) \subset Y^{*}$ (by the strictshapedness).

Appendix. A localization lemma. The proof of Remark 5.8 relied on Corollary A.3 and Proposition A.4 below. We prove first the following generalization of [9, Lemma 4.5].

LeMma A.1. Let $X$ be a compact metrizable space and let $A$ be a closed linear subspace of $C_{\mathbf{C}}(X)$. Assume that $A$ contains constant functions and separates points of $X$. Then, for every $u \in \operatorname{usc}(X)$ and $\delta>0$, there exists $\rho \in A$ and $x_{0} \in X$, such that

$$
\begin{aligned}
& u\left(x_{0}\right)+\operatorname{Re} \rho\left(x_{0}\right)>u(x)+\operatorname{Re} \rho(x), \quad x \in X \backslash\left\{x_{0}\right\}, \\
& \max |\rho|<\delta
\end{aligned}
$$

Proof. Since $A$ is separable, one can choose a countable, linearly dense subset $\left(f_{n}\right)$ of $A$, such that $\left\|f_{n}\right\| \leq 2^{-n}$. Functions $\left(f_{n}\right)$ must separate points of $X$, and so the map $F: X \rightarrow l^{2}$, where $F(x)=$ $\left(f_{1}(x), f_{2}(x), \ldots\right)$, is a homeomorphism of $X$ onto a compact subset, say $Y$, of $l^{2}$. Let $u_{0}(y)=u\left(F^{-1}(u)\right), y \in Y$. Clearly, $u_{0} \in \operatorname{usc}(Y)$. Let $u_{\varepsilon}(y)=u_{0}(y)+\varepsilon(y, y), y \in Y$, for $\varepsilon>0$. Fix $\varepsilon$ and choose $y_{0} \in Y$, such that $u_{\varepsilon}\left(y_{0}\right)=\max u_{\varepsilon}$. Let $\tilde{u}(y)=u_{\varepsilon}(y)-\varepsilon\left|y-y_{0}\right|^{2}$. Then

$$
\tilde{u}\left(y_{0}\right)>\tilde{u}(y), \quad y \in Y \backslash\left\{y_{0}\right\} \text {. }
$$

On the other hand, $\tilde{u}(y)=u_{0}(y)+2 \varepsilon \operatorname{Re}(y, y)-\varepsilon\left|y_{0}\right|^{2}$. Using this and (A.3), and letting $y=F(x)$ and $x_{0}=F^{-1}\left(y_{0}\right)$, we get $u_{0}\left(F\left(x_{0}\right)\right)+\varepsilon\left|y_{0}\right|^{2}>u_{0}(F(x))+2 \varepsilon \operatorname{Re}\left(F(x), y_{0}\right)-\varepsilon\left|y_{0}\right|^{2}$, for $x \neq x_{0}$, and so

$$
u\left(x_{0}\right)>u(x)+2 \varepsilon \operatorname{Re}\left(F(x), y_{0}\right)-2 \varepsilon\left|y_{0}\right|^{2}, \quad x \neq x_{0} .
$$

If $y_{0}=\left(y_{n}\right)$, let

$$
\rho(x)=2 \varepsilon\left(F(x), y_{0}\right)-2 \varepsilon\left|y_{0}\right|^{2}=-2 \varepsilon\left|y_{0}\right|^{2}+2 \varepsilon \sum_{n=1}^{\infty} \bar{y}_{n} f_{n}(x) .
$$

Clearly, $\rho \in A$ and satisfies (A.1), (A.2), for suitably chosen $\varepsilon$.

REMARK A.2. If $P$ is a class of functions on $R^{N}$, satisfying condition (0.1), the above lemma implies that the localization axiom (1.8) must hold. Just apply the lemma to $A=$ closed subspace of $C(K)$ consisting of restrictions to $K$ of affine functions on $R^{N}$. 
COROLlARY A.3. If $M$ is a complex manifold, such that globally analytic functions separate points of $M$, and $F$ is a class of usc functions on $M$, such that $F(U)+g \subset F(U \cap V)$, for $U \subset M$ and every pluriharmonic function $g: V \rightarrow R$, then $F$ satisfies the localization axiom (1.8).

Proof. If $K \subset M$ is as in (1.8), apply Lemma A.1 to $A=$ the uniform algebra $C(\bar{V}) \cap M(V)$, where $V$ is a relatively compact neighborhood of $K$.

Proposition A.4. Let $F$ be a class of usc functions on $M$ satisfying axioms (1.5) and (1.8). Let $K, L$ be compact sets and $u \in \operatorname{usc}(K)$, $v \in \operatorname{usc}(L)$ be such that $L \subset K \subset M, u(x) \geq v(x)$ for $x \in L$, and $\max u>\max v$. Then there is $\rho \in F(\operatorname{nbhd} K)$ and $x_{0} \in K$, such that

$$
\begin{aligned}
& (u+\rho)\left(x_{0}\right)=0>(u+\rho)(x), \quad x \in K \backslash\left\{x_{0}\right\}, \\
& \max (v+\rho) \mid L<0 .
\end{aligned}
$$

Proof. Choose $\varepsilon>0$, so that $\max u>2 \varepsilon+\max v$. By (1.8), there are $\rho_{1} \in F(\operatorname{nbhd} K)$ and such that $\left\|\rho_{1}\right\|_{K}<\varepsilon$ and $\left(u+\rho_{1}\right)\left(x_{0}\right)>$ $\left(u+\rho_{1}\right)(x)$ for $x \in K \backslash\left\{x_{0}\right\}$. Let $\rho(x)=\rho_{1}(x)-\left(u+\rho_{1}\right)\left(x_{0}\right)$. By (1.5), $\rho \in A F($ nbhd $K)$. Clearly, (A.4) holds. Furthermore, $\max \left(u+\rho_{1}\right)>$ $(\max u)-\varepsilon>\varepsilon+\max v>\max \left(v+\rho_{1}\right)$, which implies (A.5).

ReMARK A.5. Applying Lemma A.1 to a separable uniform algebra $A \subset C\left(M_{A}\right)$, one can observe that, if $F$ is a class of usc functions on $M_{A}$, such that $\operatorname{Re} A \subset A F$ (cf. Definition 1.3), then $F$ satisfies axiom (1.8). This is true, in particular, in the case of the class of $A$-subharmonic functions, studied by Gamelin and Sibony [3].

\section{REFERENCES}

[1] R. Coifman, M. Cwikel, R. Rochberg, Y. Sagher and G. Weiss, The Complex Method for Interpolation of Operators Acting on Families of Banach Spaces, Lecture Notes in Math., Vol. 779, pp. 123-153, Springer-Verlag, Berlin and New York, 1980.

[2] _ A theory of complex interpolation for families of Banach Spaces, Adv. in Math., 33 (1982), 203-229.

[3] T. W. Gamelin and N. Sibony, Subharmonicity for uniform algebras, J. Funct. Anal., 35 (1980), 64-108. 
[4] R. L. Hunt and J. J. Murray, q-Plurisubharmonic functions and a generalized Dirichlet problem, Michigan Math. J., 25 (1978), 299-316.

[5] S. G. Krantz, Function Theory of Several Complex Variables, John Wiley and Sons, New York 1982.

[6] T. Ransford, Interpolation and extrapolation of analytic multivalued functions, Proc. London Math. Soc., (3) 50 (1985), 480-504.

[7] C. E. Rickart, Natural Function Algebras, Universitext, Springer-Verlag, BerlinHeidelberg-New York 1979.

[8] Z. Slodkowski, The Bremermann-Dirichlet problem for q-plurisubharmonic functions, Ann. Scuola Norm. Sup. (Pisa), Ser. IV, vol. XI (1984), 303-326.

[9] Local maximum property and q-plurisubharmonic functions in uniform algebras, J. Math. Anal. Appl., 115 (1986), 105-130.

[10] _ Complex interpolation families of normed spaces over several-dimensional parameter space, Abstract of the Special Session in Several Complex Variables, 826th Meeting of the A.M.S., Indianapolis, April 1986.

[11] _-, Pseudoconvex classes of functions, II.

[12] _ Pseudoconvex classes of functions, III., Trans. Amer. Math. Soc., (to appear).

[13] Complex interpolation of normed and quasi-normed spaces in several dimensions, I. Trans Amer. Math. Soc., (to appear).

[14] _ Pseudoconvex classes of functions, IV.

[15] J. Wermer, Maximum modulus algebras and singularity sets, Proc. Roy. Soc. Edinburgh. Sect. A, 86 (1980), 327-331.

[16] M. Wu, On certain Kahler manifolds which are q-complete, Proc. Symp. in Pure Math., 41 (1984), 253-276.

Received April 7, 1987 and in revised form September 11, 1987.

UNIVERSITY OF ILLINOIS

ChICAGo, IL 60680 


\section{EDITORS}

\author{
V. S. VARADARAJAN \\ (Managing Editor) \\ University of California \\ Los Angeles, CA 90024 \\ HeRBert Clemens \\ University of Utah \\ Salt Lake City, UT 84112 \\ R. FINN \\ Stanford University \\ Stanford, CA 94305
}

\author{
HERMANN FLASCHKA \\ University of Arizona \\ Tucson, AZ 85721
}

RAMESh A. GANGOLLI University of Washington Seattle, WA 98195

VAUGHAN F. R. JONES University of California

Berkeley, CA 94720
ROBION KIRBY

University of California

Berkeley, CA 94720

C. C. MOORE

University of California

Berkeley, CA 94720

HAROLD STARK

University of California, San Diego

La Jolla, CA 92093

\section{ASSOCIATE EDITORS}
R. ARENS
E. F. BECKENBACH
B. H. NEUMANN
F. WOLF
K. YOSHIDA

(1906-1982)

\section{SUPPORTING INSTITUTIONS}

UNIVERSITY OF ARIZONA
UNIVERSITY OF BRITISH COLUMBIA
CALIFORNIA INSTITUTE OF TECHNOLOGY
UNIVERSITY OF CALIFORNIA
MONTANA STATE UNIVERSITY
UNIVERSITY OF NEVADA, RENO
NEW MEXICO STATE UNIVERSITY
OREGON STATE UNIVERSITY

UNIVERSITY OF ARIZONA

UNIVERSITY OF BRITISH COLUMBIA

UNIVERSITY OF CALIFORNIA

MONTANA STATE UNIVERSITY

NEW MEXICO STATE UNIVERSITY

OREGON STATE UNIVERSITY

\author{
UNIVERSITY OF OREGON \\ UNIVERSITY OF SOUTHERN CALIFORNIA \\ STANFORD UNIVERSITY \\ UNIVERSITY OF HAWAII \\ UNIVERSITY OF TOKYO \\ UNIVERSITY OF UTAH \\ WASHINGTON STATE UNIVERSITY \\ UNIVERSITY OF WASHINGTON
}

The Supporting Institutions listed above contribute to the cost of publication of this Journal, but they are not owners or publishers and have no responsibility for its content or policies.

Mathematical papers intended for publication in the Pacific Journal of Mathematics should be in typed form or offset-reproduced (not dittoed), double spaced with large margins. Please do not use built up fractions in the text of the manuscript. However, you may use them in the displayed equations. Underline Greek letters in red, German in green, and script in blue. The first paragraph must be capable of being used separately as a synopsis of the entire paper. In particular it should contain no bibliographic references. Please propose a heading for the odd numbered pages of less than 35 characters. Manuscripts, in triplicate, may be sent to any one of the editors. Please classify according to the scheme of Math. Reviews, Index to Vol. 39. Supply name and address of author to whom proofs should be sent. All other communications should be addressed to the managing editor, or Elaine Barth, University of California, Los Angeles, California 90024.

There are page-charges associated with articles appearing in the Pacific Journal of Mathematics. These charges are expected to be paid by the author's University, Government Agency or Company. If the author or authors do not have access to such Institutional support these charges are waived. Single authors will receive 50 free reprints; joint authors will receive a total of 100 free reprints. Additional copies may be obtained at cost in multiples of 50 .

The Pacific Journal of Mathematics is issued monthly as of January 1966. Regular subscription rate: $\$ 190.00$ a year (5 Vols., 10 issues). Special rate: $\$ 95.00$ a year to individual members of supporting institutions.

Subscriptions, orders for numbers issued in the last three calendar years, and changes of address should be sent to Pacific Journal of Mathematics, P.O. Box 969, Carmel Valley, CA 93924, U.S.A. Old back numbers obtainable from Kraus Periodicals Co., Route 100, Millwood, NY 10546.

The Pacific Journal of Mathematics at P.O. Box 969, Carmel Valley, CA 93924 (ISSN 0030-8730) publishes 5 volumes per year. Application to mail at Second-class postage rates is pending at Carmel Valley, California, and additional mailing offices. Postmaster: send address changes to Pacific Journal of Mathematics, P.O. Box 969, Carmel Valley, CA 93924.

\section{PUBLISHED BY PACIFIC JOURNAL OF MATHEMATICS, A NON-PROFIT CORPORATION} Copyright (c) 1988 by Pacific Journal of Mathematics 


\section{Pacific Journal of Mathematics}

Vol. 134, No. $2 \quad$ June, 1988

P. D. Allenby and M. Sears, Extension of flows via discontinuous functions

Arthur William Apter and Moti Gitik, Some results on Specker's

problem

Shiu-Yuen Cheng and Johan Tysk, An index characterization of the catenoid and index bounds for minimal surfaces in $\mathbf{R}^{4}$

Mikihiro Hayashi and Mitsuru Nakai, Point separation by bounded analytic functions of a covering Riemann surface

Charles Philip Lanski, Differential identities, Lie ideals, and Posner's theorems

Erich Miersemann, Asymptotic expansion at a corner for the capillary problem

Dietrich W. Paul, Theory of bounded groups and their bounded cohomology

Ibrahim Salama, Topological entropy and recurrence of countable chains

Zbigniew Slodkowski, Pseudoconvex classes of functions. I. Pseudoconcave

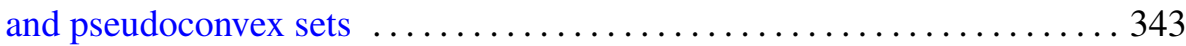

Alfons Van Daele, $K$-theory for graded Banach algebras. II . . . . . . . . . . 377

Edwin Wolf, Functions in $R^{2}(E)$ and points of the fine interior 393 